\title{
Variational Principle in an Expanding Universe
}

\author{
Giovanni Guido', Gianluigi Filippelli² \\ ${ }^{1}$ Department of Physics and Mathematics, High Scholl "C. Cavalleri" Parabiago, Milano, Italy \\ ${ }^{2}$ Astronomic Observatory "Brera", Milan, Italy \\ Email: gioguido54@gmail.com, gianluigi.filippelli@brera.inaf.it
}

How to cite this paper: Guido, G. and Filippelli, G. (2018) Variational Principle in an Expanding Universe. Journal of High Energy Physics, Gravitation and Cosmology, 4, 179-222.

https://doi.org/10.4236/jhepgc.2018.41016

Received: October 24, 2017

Accepted: January 28, 2018

Published: January 31, 2018

Copyright $\odot 2018$ by authors and Scientific Research Publishing Inc. This work is licensed under the Creative Commons Attribution International License (CC BY 4.0).

http://creativecommons.org/licenses/by/4.0/

\begin{abstract}
In this paper we complete the relativistic cosmological theory because we extend the variational principle including variations of metric induced by expansion of the space. We will show that the mass not only curves Space and Time but also generates them: we'll speak of the principle of mass-space equivalence. Then the increasing mass generates variations of metric as also the space increasing or expansion. So, the dark component of the mass generates additional gravity around galaxies as well as additional space, which generates a pressure (dark energy) accelerating the galaxies in their move away. All this could explain the cosmic coincidence $\left(\Omega_{\mathrm{dm}} / \Omega_{\mathrm{de}} \approx 1\right)$. To talk about the increasing mass is equivalent to speak of mass creation in universe, causing the variation of the tensor ( $T$ ) mass-energy tensor of all component fields. We conjecture that its variation is caused by mass-energy flow comes out from a physical system $(\Theta)$ composed by set of uncoupled quantum oscillators (structure of no-field) in vacuum state. All this allows formulating a variational principle which generates the cosmological equation with the $(\Lambda)$ parameter and a tensor $T^{*}$ with variable mass density, where $T^{*}(T, \Theta)$.
\end{abstract}

\section{Keywords}

Mass-Space, Lattice-Field, No-Field, Intrinsic Quantum Oscillator, Dark Matter, Dark Energy, Vacuum

\section{Introduction}

In modern cosmology the expansion of space is not derived from any fundamental principle of physics but is defined as empirical properties of space in the universe. In fact, one notes that the universe expansion is detected to be a mutual motion away between galaxies. In literature this can be understood as consequence of any "stretching" of a whatever spatial length, which can be described 
by parameter $a(t)$, you see Friedman's solutions. Always in literature there are some hypotheses about the origin of the expansion but these studies lead to problematic aspects and frequently in contrast to General Relativity (GR), which is the theory of base of a modern cosmology coherent with astronomical observations.

In the relativistic cosmologies, we can well known the topics concerning the cosmic balance in a universe governed by gravitation: to avoid a rapid gravitational collapse, which would deny also our existence itself, Einstein puts "ad hoc", but without identifying cause, the existence of a balancing pressure "antigravity", expressed by the constant $(\Lambda)$ introduced into the relativistic cosmology equation. When Friedmann found the solutions of these (without the constant $(\Lambda)$ ) which not including the gravitational collapse, Einstein breathed a sigh of relief: introducing into space a scale factor $a(t)$ dilating galactic distances, you could avoid the gravitational collapse in an expanding universe with properties of homogeneity and isotropy. When Hubble then declared unequivocally the galaxies away from each other, Einstein defined $(\Lambda)$ the biggest error of his life.

Nevertheless, to explain the expansion through only the $a(t)$ scale factor it could result analogous to take the "blind alley" of the tautology: $a(t)$ is rationally admissible if we admit the expansion and vice versa. So the $a(t)$ scale factor would result, equally of $(\Lambda)$, you see Einstein, a mathematical artifice introduced "ad hoc".

This "tautology" can be avoided only if we resort to something else: as it has been done for the gravitation, it is necessary to express the expansion through an intrinsic property of the space and matter, coexisting and inseparable: it makes no sense talking about space in the universe if there is not matter. Einstein stopped instead to the conception that the mass influences the (ST) Space-Time (or the metric) which we use for representation of physical phenomenon. The gravity is so the expression of this "influence": but this aspect negates that the ST is only a mere our representation where placing the physical objects. If to gravity we associate a "field" of perturbation of the ST built and used by us, then the ST must become a physical object with some well-defined properties: it must become a "field". Thus, the mass becomes (par. 2.1) the source charge of a "Space-Time field" and of its perturbation (gravitational field), to the same way of the electric charge, rising of electromagnetic Field (EMF) and of its perturbation. The mass so should become rising of Space and Time in the universe, thus we can talk about universe as Space-Time field. In fact, if the universe (U) is thought being as "all particles-fields" then all particles are built on the Space-Time Field (we will indicate this as "hypothesis of structure"). In describing the whole universe we feel then there to support, in order to achieve the completeness of the GR, that gravitation and the expansion of the universe must be included among the properties of the $\mathrm{S}$-T field (or metric field). In fact, if we take into account the universe expansion and the acceleration of galaxies, we can assert [1] that the universe seems to be a unique S-T field which carries out two important properties: on the one hand its massive components (ordinary mass and dark) and its gravitational field are "aggregating", while the other component, that spatial, is "expanding" (expansion and 
its acceleration). Not only but the two properties, indicated above, should be expressed in the context of a unique variational principle. Built a theory based on a unique variational principle we adopt the property [1] that the mass generates space (par. 2.2) and thus the expansion can be caused by "mass creation" in universe. We'll say that the mass creation generates space (expansion and acceleration) and creates time (evolution and cosmic time) as also, as it is well known, the perturbations of Space-Time (ST), you see the gravitational field $\Leftrightarrow$ ST metric. We speak on the creation space because, you see the Compton wave length $\left(\lambda_{c}\right),\left[\mathrm{m} \Leftrightarrow \lambda_{c}\right]$; then, one builds the idea of "lattice-field" (par. 2.2) associated to set of particles with mass $\left(m_{i}\right)$ and space-step $\left(\lambda_{i}\right)$. We will talk about "increasing mass-space" in lattice-fields with mass $\left(m_{i}\right)$ or also said "Universe-lattices" $U_{i}$, with $\left[U_{i} \subset U\right.$ ]. Then we bring back synthetically that already treated in [1] where the idea of a Universe increasing (par. 2.3) in mass-space and satisfying the Hubble law is made. We make note that if the universe expansion is detected to be a mutual spacing between galaxies, then, there is a physical equivalence between showing that this is caused by an increasing "quantity" of space interposed between them or that the expansion is a "stretching" of the space. Besides, asserting that adding space inside space is equivalent to creating additional space we can describe also the acceleration of expansion: we show, in fact, (par. 2.4) that in any $U_{i}$ universe-lattice it is possible obtain a generalization of Hubble law describing also an acceleration of space.

In par. 3.1, synthetically, we show the theory of a "Universe at lattice-Fields" developed in the article [1]. These lattices overlap and intersect between themselves, originating some evolutionary phase of Universe, with descriptive parameters coincident to some astronomic dates, as the duration time of Nucleosynthesis phase and that at Hydrogen (from recombination today coincident with universe time). The present universe is so the Hydrogen Universe with critic density (flat metric) very more mayor of that ordinary matter, opening so the road to hypothesis of dark matter. Building (par. 3.1) the intersection between the dark lattice and Baryonic lattice $\left(U_{d} \cap U_{B}\right)$ ) one determines the mass density with value next to that astronomic. In par. 3.2 we show that the theory of the lattices of field at massive coupling is coherent with the EP and can solely express a relativistic cosmology. Besides correlating the mass to space this theory "completes" the GR rather "extends" it: the mass acts on the space in "totus", because the mass curves the space and produces it. The "Universe at lattice-Fields" (ULF) resolves some problematic of the modern cosmology and (par. 3.2) gives some possible future developments of our universe as the one which describes a universe reaching the flat phase after once of 14.82 billions of years from the Big Bang and then it should pass to a metrics closed with prevalence of the gravitational force. So it is admitted the possibility that to the end of the contraction phase there will is a "Big Crunch". Nothing prohibits then another new Big Bang and therefore a new universe: this makes a "Cyclical Universe".

For give a rigorous mathematical base to the theory of the "Universe at lat- 
tice-fields" (ULF) we begin (par. 4.1) with a critical review of the Einstein-Friedmann (EF) cosmological model. We go highlighting the possible solution to its incompleteness (or the (EF) model does not describes in "totus" the object "Universe-(Space-Time)"), showing that the completeness is achieved if we include the expansion as a property of the Mass-Space in accretion. First of all we go showing the full compatibility of this idea (the "increasing mass-space") with the theory of the GR. As is well known source amending the metrics of space is the mass, therefore one can discuss about mass becoming the source acting on the space by increasing its size, as if it "would create" space or as if it "would add" an ulterior additional space. We are so in front of the possibility of extending our way of seeing the relation [Mass $\Leftrightarrow$ Space] that would allow us to explain both the expansion that acceleration galactic. In fact, if to expansion we associate a space flow then in order to the mass-space equivalence we have added a mass flow which makes a metric variation and produces an additional pressure. Then we prove (4.2) that the galactic acceleration is just the effect of an additional pressure consequent to the creation of mass-space, which will expressed by a parameter $(\Lambda)$. Nevertheless (par. 4.3) the mass-space flow push us to admit the existence of his source which we place in a physical system $(\Theta)$ coexisting to the physical system of all fields of the Universe (U-universe Field) but from it "separate" or it not include in tensor mass-energy of all fields. Nevertheless, if we want to fall in the metaphysics then we have to consider both $U$-field and $(\Theta)$ as two different states of an only physical system $\left(\mathrm{U}^{*}\right)$. This "physical state", separated to all fields, cannot be a "field": if the field is elastically built as a set coupled quantum oscillators then we have to admit a physical state of oscillators no-coupled. If $U$ is the set of all field-particles then $(\Theta)$ is the set of quantum oscillators uncoupled [1]: this is a notable new. To understand this then we resort to a new representation of quantum oscillator which can admit a vacuum state of uncoupled quantum oscillators. In [2] [3], one represents a quantum oscillator composited by two sub-oscillators with semi-quanta (IQuO). The vacuum state of a IQuO uncoupled is different by that of the "classic" quantum oscillator and allows the existence of an "isolated" oscillator. The IQuO-representation so allows the existence of physical system composed only by set sub-oscillators in vacuum state and uncoupled $(\Theta)$. Therefore the evolution of the universe would be consequences of a continuous exchange of quanta flow of energy between these two systems ( $U$, $\Theta)$.

The decanting of quanta from $\Theta$ to $U$ happens "wherever" in $U$ (this because $\Theta$ is "underground" in $\left.\left(\Xi_{U}\right)\right)$ for obtain a homogeny and isotropic expansion. A possibility of decanting could be the one a sort of "gate" placed in way homogenous "wherever" in U, like the galactic black holes. Remember that on $\left(\Xi_{U}\right)$ there are structured two form of mater: the dark mass $\left(\underline{\Xi}_{D}\right)$, which is a base mass no-radiant, and the ordinary mass $\left(\underline{\Xi}_{O}\right)$, radiant structure forms (particles of standard model). Then we have admit that a portion of flow of energy (quanta) going from $(\Theta)$ to Universe $(U)$ origins the dark matter. 
But, in order to "mass-space" equivalence, these asserts lead us to speak the mass-space flow coming from $(\Theta)$ gives rise also to mass-energy dark $\left[\underline{\Xi}_{\mathrm{Dm}}\right.$, $\left.\underline{\Xi}_{\mathrm{De}}\right]$, where $\left(\underline{\Xi}_{\mathrm{De}}\right)$ is the space field making the galactic acceleration. This is a very important news and original.

Note that the generalized law of Hubble (expansion and acceleration) is drawn in a universe to mass-space increasing (ordinary massive fields and dark massive fields), where the splitting of the IQuO happens in everybody the points of the space (see par. 2.4). This is possible if we consider the ordinary matter generating the lattices of increasing mass built on fundamental particles that make up the universe and fill it. Being an increasing mass lattices are also increasing in space, hence the expansion and the "ordinary" Hubble law. Instead in order to the dark matter, we could think that this can be the cause of the additional pressure: we make the hypothesis that it could be due to mass-space flow coming out from the Black Holes, placed in way homogenous "wherever" in U (see galaxies). The equivalence mass-Space pushes us to admit after that there is an equivalence between dark mass flow and space flow (pressure of dark energy) and this should determines that $\left(\Omega_{\mathrm{dm}} / \Omega_{\mathrm{de}} \approx 1\right)$.

Going forth we will understand $(\Theta)$ as a "perfect fluid" contained into a "inert underground container" into Universe which come out (as mass-space flow)

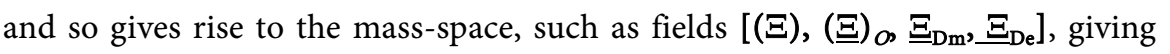
rise to the space increase, which is observed experimentally as galactic expansion and additional pressure that accelerates galaxies.

It is clear then that we can substitute the initial singularity of the Big Bang with the not-field $\Theta$. The cosmic time so has had origin when the quanta flow (for the coupling of quantum oscillator) from $\Theta$ in $T$ has had the origin, as well as the space.

All this allows elaborating (par. 5.1 and par. 5.2) a variational principle taking in account of expansion which is the origin of variations of the tensor $g_{i k}$ (one remembers that the curvature ray increases to dilating of the curve). Elaborating the action function $S_{g}$ in the variational principle we derive the form of G Einstein tensor with the $(\Lambda)$ parameter. In order to equivalence mass-space we note after that also the mass-energy tensor $T_{i k}$ has a mass density variable. That is possible if at physical state $U$ (the Universe), seen as set of particles-fields, we add a physical state $(\Theta)$, seen as "inert" reservoir of energy quanta, with which $U$ changes energy (mass-space flow). All this allows deriving the cosmological equations with the $(\Lambda)$ parameter, which describe a universe with variable metric perfectly "compatible" with the RG.

The cosmological theory that emerges thus proves to be a completion of the EF model because it completes the vision of the Universe $\left(U \rightarrow U^{\star}=U+\Theta\right)$ and incorporates this it into a theory to wider horizon as is that of Space-Time Field.

This theory, perfectly consistent with the theory of relativity and quantum mechanics, can well be included among the extended theories of gravity, listed and synthesized in the article of Corda (Int. J. Mod. Phys. D, 18, 2275 (2009)). 
Nevertheless, a more accurate look allows us to see it as "more" original for its bold starting hypothesis, built on the innovative idea of quantum oscillator at semi-quanta (IQuO). Building fields with IQuO oscillators implies having more degrees of freedom that explains the origin of the electric charge and the mass and other. If we consider IQuO oscillators in a vacuum state, there is the possibility also to build a new physical state of vacuum on which it is possible to build a physical energy system but "inert" and therefore not computable in the energy expressed by the tensor $\mathrm{T}$ of cosmologic equation. All this allows seeing the universe through an unseen vision capable of deeply understanding how all these fundamental physical phenomena take evolution. You see its expansion and acceleration, as well as dark matter-energy.

\section{The Mass-Space of the Universe}

\subsection{The Nature of the Mass}

In a study precedent [1] we made note that the Theory of Relativity establishes a deep physical connection between space, time and material objects (particles-fields): we cannot describe particles and their interactions without referring to space and time, and, at the other hand, the space and time haven't a physical sense without objects (particles), in fact remember that a reference frame (RF) be built with material objects reciprocity at rest. Therefore, one could assert that if particles are to be considered like an objective reality, then also time and space, used for describe particles and their interactions, should be objectively considered as intrinsic "real characteristics" of the particles. So like we stated that the particles are in the Space-Time (ST) and to same way we will say that the "Space-Time is into particles". Remember that an elementary particle (i.e. electron) is seen in relativity theory like a point in (ST) of any reference frame while in quantum theory it is seen like a field $(\Psi)$. The characteristic of field into particle is detected in his dual behavior (undulatory in $\Psi$ and corpuscular in the quanta). If we associate a wave behavior to particle (you see the diffraction with electrons and photons) it is permissible to conjecture that "something" oscillates "inside" a particle: we talk about internal "clock" with proper frequency $\left(\omega_{0}\right)$. It's evident that the frequency $\left(\omega_{0}\right)$ generates the "proper time" $(\tau)$ of massive particle.

Then we conjectured that the mass is the physical expression of the proper frequency [mass $\left(m_{0}\right) \Leftrightarrow$ frequency $\left(\omega_{0}\right)$ ] related to a particular elastic coupling between the oscillators of a scalar field $(\Xi)$ of base. We don't have to think of associating a scalar field $\Xi_{i}$ to any i-particle but a unique scalar of background of the universe: in this way we can say that the Universe is also a scalar field $(\Xi)$, you see the definition that Universe is "all of this that is". Then $(\Xi)$ is a massless scalar field which exists in whole Universe (pointed with $\Xi_{U}$ ) and which is made by a lattice of 1-dim. Chains of Quantum Oscillators (IQuO) reciprocally coupled analogous to a set of beads connected through springs (see Figure 1):

The conjecture [mass $\Leftrightarrow$ frequency] is derived by equivalence between two 
equations:

$$
\left\{E^{2}=m^{2} c^{4}+p^{2} c^{2} \Leftrightarrow \omega^{2}=\omega_{0}^{2}+k^{2} c^{2}\right\}
$$

The first equation is the famous equation of the relativity for massive particle which are described by Klein-Gordon equation; the second equation describes a set of pendulums coupled through springs which are described the same by a same Klein-Gordon equation with the following substitutions: [ $(E \rightarrow \omega),\left(m \rightarrow \omega_{0}\right),(p$ $\rightarrow k)$ ]. Then [1] we conjectured that the mass is given by a particular "transversal additional coupling" between the oscillator chains of the base $\boldsymbol{\Xi}$-Field, as the one present between the pendulums, because the two systems are analogous (see Figure 2).

If the second equation (in 2.1) is the dispersion relation of the Klein-Gordon equation of pendulums coupled; at same way the first equation is the dispersion relation of the Klein-Gordon equation of massive particles. The frequency $\left(\omega_{0}\right)$ generates so the proper mass $\left(m_{0}\right):\left[m_{0}=\hbar \omega_{0} / c^{2}\right]$.

Following this relation, we conjectured that the mass $\left(m_{0}\right)$ is the physical expression of the proper frequency $\left(\omega_{0}\right)$ related to a particular elastic coupling

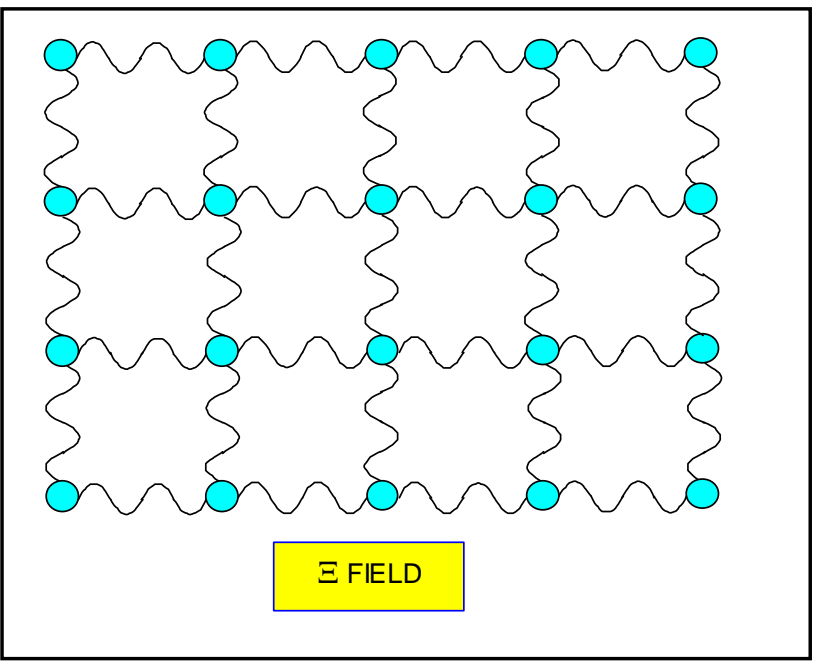

Figure 1. Field equivalent to set of beads with springs.

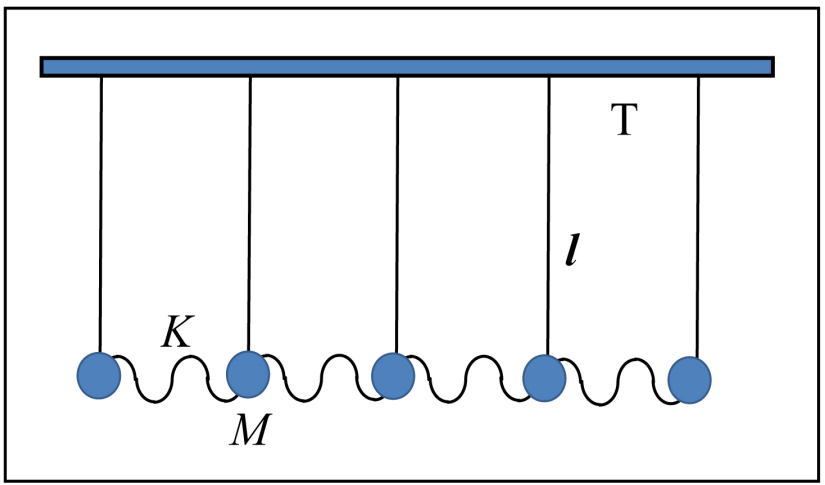

Figure 2. Set of pendulums coupled through springs. 
which is additional to the one already existing between the oscillators of massless scalar field. This "additional coupling" which produces the mass in a scalar field (as $\Xi$ ) has been pointed out as "massive coupling". We think of the Compton wave length $\left(\lambda_{c}\right)$ associated to any massive particle: $\left[\lambda_{0}=\hbar / m c\right]$. In this way we can build a massive field $\left(\Xi_{i}\right)$ with lattice structure on the $(\Xi)$ field, where is given a “Time Step" $\left(\tau_{i}\right)$ and a "Space Step" $\left(\lambda_{\mathrm{i}}\right)$; note that on this lattice structure $\left(\Xi_{i}\right)$ it is possible to build a Reference Frame (RF) associated with a massive particle $\left(m_{i}\right)$ and with Compton wavelength $\left(\lambda_{i}\right)$. In this way, the mass $\left(m_{i}\right)$ of each particle could be identified by a distinct and articulated structure of coupling of Quantum Oscillators of the $\Xi$-field [3a]. We indicated this as "Structure Hypothesis" of massive particles in $\Xi_{U}$ field. Following the Standard Model and the structure hypothesis we could suppose that the basic fermions (electron, for leptons, and proton, viewed like quarks $\mathrm{u}, \mathrm{d}$, for baryons) can determine some processes of space-mass creation in the universe, because they are basic structures of couplings of the Quantum Oscillators cosmology.

\subsection{The Field at "Massive Lattice"}

Moreover, it has been possible to assign to a set of identical massive particles, with mass $\left(m_{i}\right)$, a field at lattice structure $\left(\Xi_{i}\right)$ which we have called "lattice-field" of universe or "universe-lattice", denoted by $U_{\dot{i}}$ In this way the mass express the additional coupling between chains of oscillators of a quantum field, building so a "lattice shaped field" or "massive lattice" (see Figure 3).

We noted that on this lattice structure $\left(\Xi_{i}\right)$ it is possible to build a $\left(\Sigma_{0}\right)$ Reference Frame (RF) with a "motion" in the proper time $\left(\tau_{0}\right)$ and a spatial dimension $\left(\lambda_{0}\right)$ : we shall say that the "Space-Time is into particles". We can so consider the universe as set $\left(\Sigma_{U}\right)$ of the local reference frames $\left(\Sigma_{0}\right)$, associated with massive particles.

The symmetry group of the universe based on the lattices is the Poincare group.

We note firstly that the base field $\left(\Xi_{U}\right)$ itself does not express any additional transversal coupling that provides mass and thus we cannot define in it space

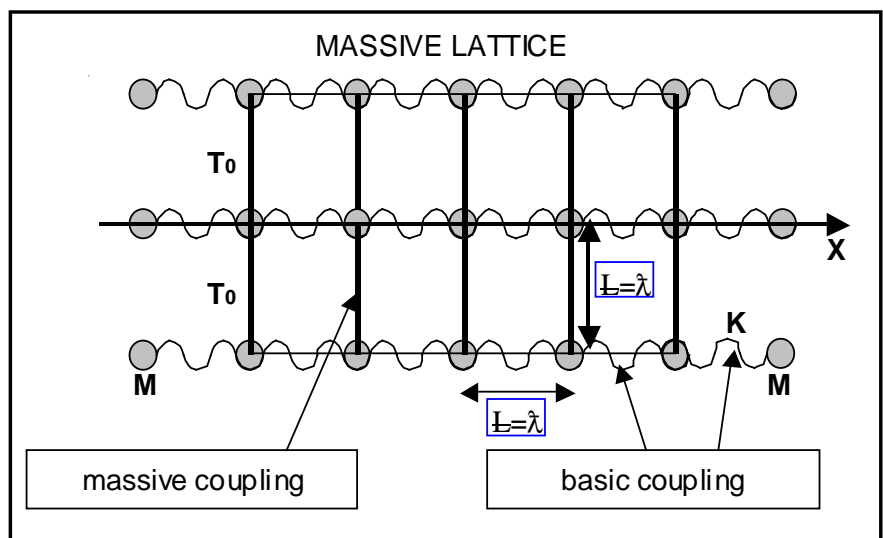

Figure 3. Massive field as lattice of "pendulums" with springs. 
and time; just only for a descriptive aspect we represent it as a lattice with "flat" metric (in metric it is $R_{i k l m}=0$ with $T_{i k}=0$ ). Still we not that the $\left(\underline{\Xi}_{i}\right.$ ) lattice of chains associated with a single and "isolated" massive particle, originated in $\left(\Xi_{U}\right)$, will be to "metric" flat while a superposition of massive lattices can generate an additional elastic tensions $\left(T^{\circ}\right)$ that "curves" the resulting lattice (you see the following Figure 4).

We realize that the super massive lattice builds a ST tissue at "curve" geometry well described in GR of Einstein [4]. The overlap of massive lattices implies the transitions: $\left[\left(R_{i j}=0 \rightarrow R_{i j}\right) ;\left(T \equiv m_{i} \rightarrow T_{i j}\right)\right] \rightarrow\left[R_{i j}=\kappa T_{i j}\right]$.

Now, we can build a whole RF for every galaxy as well as for clusters of galaxies until whole universe. The RF of the whole universe coincides with the "comovent FR" [5] defined by actual cosmology, to which a proper cosmic time is associated equivalent to the age of the universe. We could associate an only and unique field of base also to each "comovent RF" in any galaxy: this will be a massive scalar field of fund which will indicate with $\left(\underline{\Xi}_{G}\right)$. The "comovent" RF coincides so with the "Space-Time lattice" of the universe which it is built on the base field $\left(\boldsymbol{\Xi}_{U}\right)$ involving a "massive couplings" $\left(m_{i}\right)$ with spatial dimensions $\left(\boldsymbol{\lambda}_{i}\right)$; besides, asserting that in the universe there is a unique cosmic time, it is physically equivalent to say that in whole universe there is a "massive field" of base $\left(\underline{\Xi}_{U}\right)$. So, the "Universe Field" will be expressed by two components: the first is $\left[\Xi_{U} \equiv\left(\Xi_{i}\right)\right]$ relatives to base $\Xi_{U}$ field and to the set of massless field lattices $\left(\Xi_{i}\right)$ (you see the photons remembering that a vector fields is the combination of scalar $\Xi$-fields or components) and, at the same time, the second is ( $\left.\underline{\Xi}_{U}\right)$, a massive field relative to unique cosmic time which any observer notes in any galaxy $\left(\underline{\Xi}_{G}\right)$. To associate a unique base field to any massive field it is not equivalent to believe that $\left(\underline{\underline{\Xi}}_{U}\right)$ is a sort of elastic ether pervading an absolute space, refuted by the theory of relativity, because the field $\left(\underline{\underline{\Xi}}_{U}\right)$ it is itself the cosmic space-time (you see the RF comovent) which an any observer of the universe builds in proper reference frame at rest. We could conjecture that the dark matter is in relation with

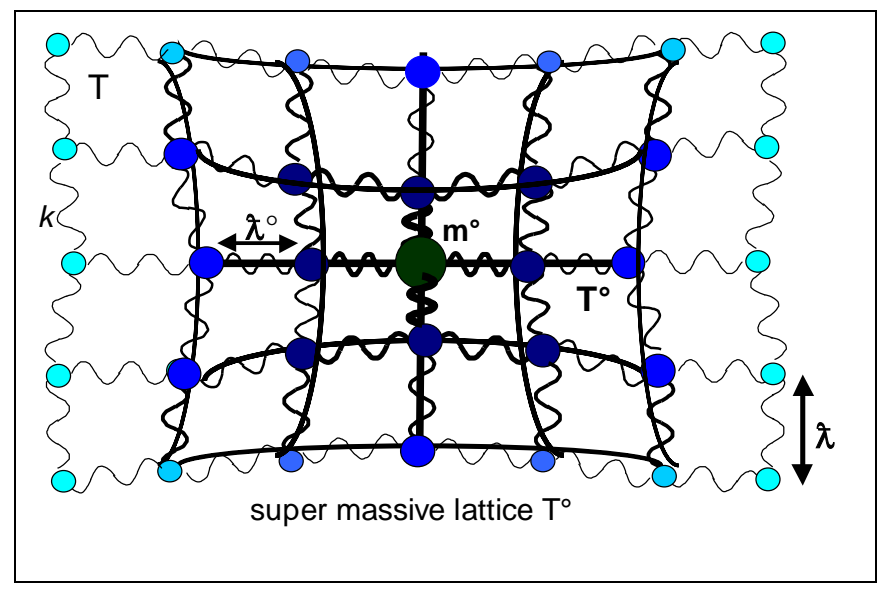

Figure 4. Super massive lattice "curved". 


$$
\left(\underline{\Xi}_{U}\right):\left[\underline{\Xi}_{U} \Leftrightarrow\left(\underline{\Xi}_{G}\right) \Leftrightarrow \underline{\Xi}_{D}\right],
$$

where $\left(\underline{\Xi}_{D}\right)$ is the field of Dark Matter (you see the halos of dark matter around any galaxy); you see also the [1], with possible lattice-fields $\left(\underline{\Xi}_{D i}\right)$.

We noted also that the relation mass-frequency implies that the mass meaning presented in our theory do no admits negative values of mass; in this way we show that do not exist antimatter associates to initially negative masses and also if these come admitted as existing the CPT transformations makes them with positive energy, you see the CPT-symmetry; in the theory of the massive lattices one demonstrates that negative Lagrangians associated to antimatter exist no which can origin a repulsion between matter and antimatter, as instead it is proposed by some physicists in opposition to General Relativity (RG). Then we showed that la present theory is instead coherent with the Equivalence Principle (EP) and then proposes a relativistic cosmology.

\subsection{The Increasing Mass-Space}

Indeed, an observer [5] cannot be "preferential" (for the invariance of the laws of physics) than other observers of the universe by detecting various or particular motions of distant galactic matter.

However, this same motion of the galaxies can only exist if every observer is at the "centre" of the universe observed.

Combining these assertions we obtain a universe in which the "apparent" relative motion of the various FR (galaxies) should be "radial" to any observer (isotropy).

This implies that an observer must see in all directions objects in distancing or, alternatively, in approaching. As already one knows, this is the enunciation of cosmological principle, of which the Hubble law is empirical demonstration.

Although one admits the consistence between first principle of relativity and cosmological principle, nevertheless the type of galactic radial motion, expansion or contraction, cannot be derived by these principle: we ask so about the origin the expansion. We could suppose [1] that the Universe expansion (or eventually contraction) is a property of a Universe "field" at increasing space: a process of continuous creation (or addition) of space between two any objects of the universe (galaxies).

In this case if the process of continuous creation (or addition) of space is homogeneous and isotropous then this process is compatible with the first principle of relativity. This is equivalent to stating that describing the expansion through the parameter of spatial dilation $a(t)$ is physically indistinguishable from consider that between any two objects that space is created. If we show that the Hubble law, compatible with the cosmological principle, can be derived from a hypothesis of increasing space for creating or adding, we demonstrate also that the expansion described by the parameter of expansion $a(t)$, is indistinguishable physically from considering that between any two objects you create space. 
For justify these assumptions we go back to Compton wave length $\left(\boldsymbol{\lambda}_{c}\right)$.

Through the definition of Compton wave length $\left(\boldsymbol{t}_{c}\right)$ and $(\tau)$, we have assert that a massive particle introduces in universe the dimensions of the space and time. Then, we have made note [1] that when a massive particle is created in $\Xi$-(lattice-field) we are forced to add space (you see the $\lambda_{i}$ ) and time $\left(\tau_{i}\right)$ in universe $\Xi_{U}$ because the particles are "space" and "time". This allow us of say that the creation of massive particles "creates" new space (see the $\lambda_{c}$ ) or adds space into base universe $\left(\underline{\underline{\Xi}}_{U}\right)$, with the mean of the Space-Time Reference Frame "comovent" of any galactic observer $\left(\underline{\underline{\Xi}}_{G}\right)$. So, we show that the origin of the expansion of the universe is to be found in a sort of "creation of space" resulting to the "mass creation" or creation of massive particles $\left(\underline{\Xi}_{i}\right)$ in various universe-lattices $U_{p}$ these built on the $\left(\underline{\Xi}_{U}\right)$. Idem, one can say about the cosmic time resulting to the "mass creation". By this asserts we can conjecture that the expansion of universe $\left(\underline{\Xi}_{U}\right)$ is the effect of a space increasing continually derived by Space creation in $\left[\left(\underline{\Xi}_{i}\right) \subset\left(\underline{\underline{\Xi}}_{U}\right)\right]$.

\subsection{The Generalized Hubble Law}

We showed [1] that in any $U_{i}$ universe-lattice the Hubble law can be derived precisely by the "creation of space" resulting by the birth of $\left(m_{i}\right)$ massive particles in $\Xi$. We will show now that a universe in increasing space quantity-with specific mode of increase-is indeed an expanding universe described by a generalized Hubble's law which can also cause acceleration between the galaxies.

At distance $(\lambda)$ we place initially (you see Figure 5) two objects $(O)$ and (A) which could be also two (IQuO) quantum oscillators (respectively $(\mathrm{O})$ and $(\mathrm{A})$ ) connected by another (IQuO) $(\mathrm{OA})$ with spatial dimension $(\lambda)$. The three (IQuO) could became the constituents of an emerging chain (space line) of the base field of the universe $\left(\Xi_{U}\right)$, built using IQuO described by the parameters $(m, \lambda)$.

Let's suppose, in $\tau$ time, that into the IQuO system [(O), (OA), (A)] a "splitting" of $(\mathrm{OA})$ (IQuO of connection) in (OB)-IQuO and (BA)-IQuO is originated; it follows that the distance between $(\mathrm{O})$ and $(\mathrm{A})$ will become the double than before: $(\mathrm{OA}=\mathrm{OB}+\mathrm{BA}=2 \lambda)$. The cause of this splitting could be a flow of "quanta" originated by a physical system $(\Theta)$, specified more ahead in this article.

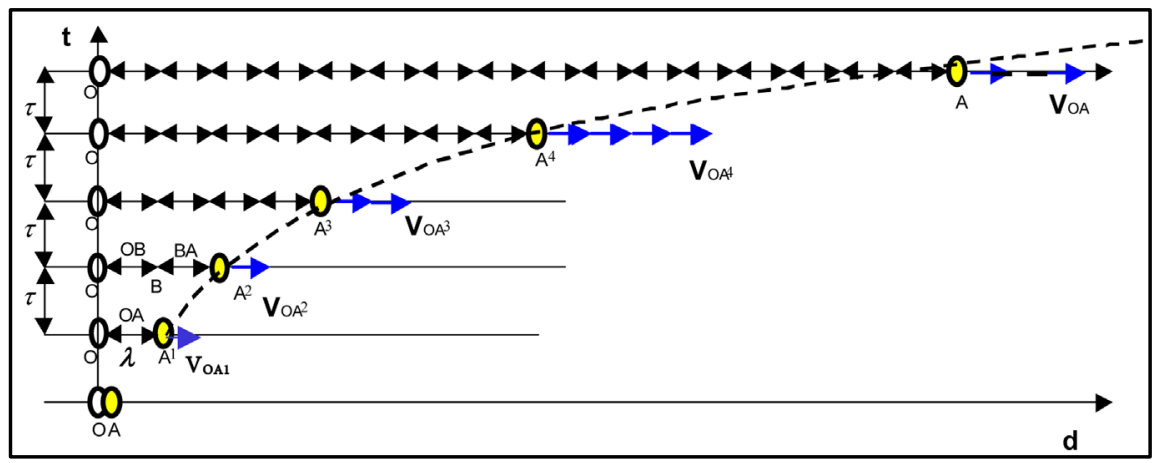

Figure 5. Elongation of the line OA of field. 
In the next time interval $\tau$ is possible further splitting of IQuO connecting (you see the Figure 5). Object (A) goes away from $(\mathrm{O})$ with recession velocity given by: $V_{\mathrm{OA}}=(\Delta \mathrm{OA}(\tau) / \tau)$, therefore $V_{\mathrm{OA} 1}=[(2 \lambda-\lambda) / \tau]=v ; V_{\mathrm{OA} 2}=[(4 \lambda-2 \lambda) / \tau]=v$.

Note that $V_{\mathrm{OA} 2}=2 V_{\mathrm{OA} 1}$ because OA2 $=2 \mathrm{OA} 1$.

The splitting process (or equivalently doubling) of any IQuO goes on in time and determines a continuum elongation of the $\Xi$ chain (you see the Figure 5), where velocity $V_{\mathrm{OA}}$ doubles to the doubling of distance.

This will involve in an expansion phenomenon (elongation of the line OA) described in Figure 6, analogous to the elongation of the line OA described mathematically by the expansion parameter $a(t)$ present in the equations of Fried$\operatorname{man}[6]$.

If we replace $(\mathrm{O}),(\mathrm{B}),(\mathrm{A})$ with galaxies then one gets an expanding universe where the recession velocity from $(\mathrm{O})$ increases with the increasing distance between them. It can be noted mathematically that the mean velocity of recession follows the law:

$$
V_{(n, 0)}=\left[\frac{2^{n} \lambda_{i}}{2 \tau(\eta)}\right]
$$

where $\left[\tau \neq \tau_{i}=(\lambda / c)\right]$.

If we set the distance $(d)$ with $\left[d(n)=(2)^{(n-1)} \lambda\right]$, the "cosmic" time with $\left[t_{U}=\right.$ $n \tau]$ and the $\left.\left[H(\eta)=1 / \tau_{\eta}\right)\right]$ where $(\eta)$ represents a parameter which can depend by evolutionist characteristics of the universe, then it is obtained a particular law of expansion:

$$
V=H(\eta) d
$$

where $H(\eta)$ is a physical quantity that represents the rate of increase of the space.

So we have showed that the generalization of Hubble law can be derived precisely by the "creation of space" resulting by the birth of $\left(\Xi_{\mathrm{i}}\right)$-massive particles in $(\Xi)$, which increase the lattice- field of $U_{i} \subset U$, with $\underline{\Xi}_{i} \in \underline{\underline{\Xi}}_{U} \equiv\left[\left(\underline{\Xi}_{O}\right)\right.$, 㰯], where $\left(\underline{\underline{\Xi}}_{O}\right)$ are the fields of ordinary matter and that of $\left(\underline{\underline{\Xi}}_{D}\right)$ dark matter.

So, describe the expansion through the parameter of spatial dilation $a(t)$ is physically indistinguishable from considering that between any two objects that

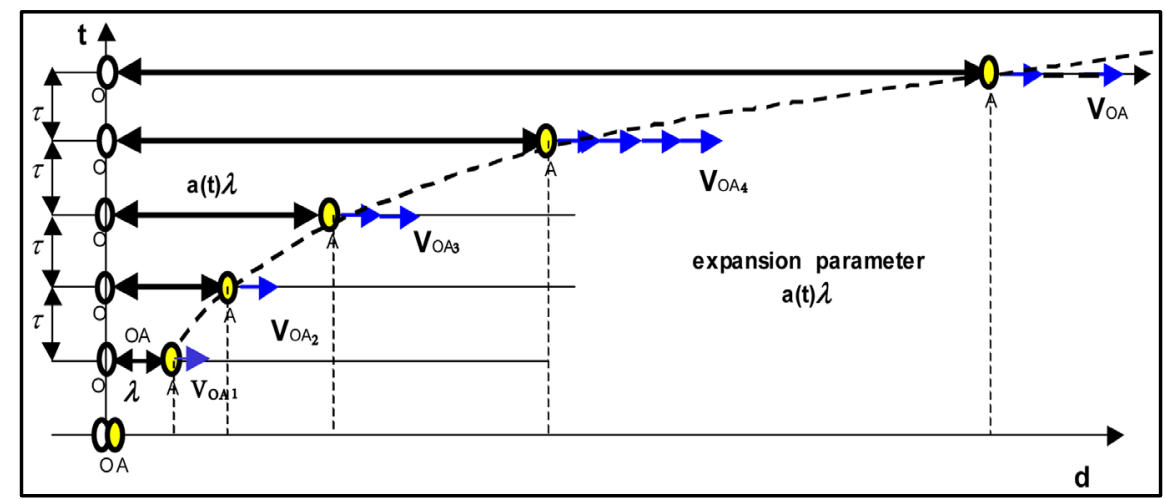

Figure 6. Expansion curve. 
space is created.

\section{The "Universe at Lattice-Fields"}

\subsection{The "Universe at Lattice-Fields"}

Speaking of equivalence mass-space we showed that a universe with "flat" metric exists only if there is a state of balance between gravity (originated by mass) and space (also originated by mass).

In [1] we specified the idea of "lattice field" present in the whole universe or Universe-lattice $U_{i}$, built on the particles of some type with mass $m_{i}$. If number (n) of massive particles increases (increasing mass), that is $\left(m_{i} \rightarrow n m_{i}\right)$, it follows that $U_{i}$ will be increasing in space to pass of time (time evolution). We proved mathematically the balancing mass-space be achieved by each lattice $U_{i}$ only at a certain moment of its evolution, where at pass of the time the $U_{i}$-lattice increases in mass (increasing number of particles) as well as in space. Thus $U_{i}$ is a universe lattice with a "variable metric", going from a value of negative curvature (open universe for future reference) with accelerated expansion to one of positive curvature (closed universe) after going through an intermediate critic phase with a critic value of metric (flat universe). It is proved that the time $\left(\tau_{c}\right)$ to reach the critical phase is connected to the coupling gravitational constant $\left(\alpha_{i}\right)$ of each $U_{i}$. After we have defined also the universe-lattice $U_{H}$ like the "intersection" between the pair of universes-lattice $\left(U_{p}, U_{e}\right)$, because it is assumed (by the standard model), that our universe $U$ is built on particulars set of base universe-lattice $U_{p}$, such as that of the electron $\left(U_{e}\right)$ for leptons and that of the nucleon $\left(U_{p}\right)$ for the baryons (quarks).

Then, we considered that the hydrogen atom builds (through the intermediation of the photons, the quanta of the electromagnetic field) the intersection between the two lattices or $\left[(U)_{(p, e)}=(U)_{H}\right]$.

This allows saying that our universe is a "Hydrogen Universe".

This aspect makes determine the factor $\left(k_{s}\right)$ which allows the "overlap" of the Compton wave lengths of two universe-lattices $\left[\left(U_{p}\right),\left(U_{e}\right)\right]$.

Then, one obtains by simple calculations that the $\left(\tau_{c}\right)$ of $(U)_{H}$ is coincident (within the errors range) with the $(\tau)$ present age of our universe, defined only by astronomical observations (you see spatial telescope Planck). This leads us to believe that our universe is now in flat metric phase because it is near to the next critical phase of a universe with a variable metric.

We have noted that the $\rho_{c}$ critical density of $(U)_{(n, e)}$, is greater than the one calculated in "nucleon masses" $\left(\rho_{n}\right)_{c}$ and this detects the need of existence of a mass not visible that it bridges gap the difference between two density (missing mass). We can analyze so the phase of Nucleosynthesis built on the universe-lattice $U_{N}$ which is intersection of two lattices of hadrons:

$$
(U)_{(\alpha, \pi)}=\left(U_{\alpha} \cap U_{\pi}\right) \equiv U_{N} .
$$

We saw that $U_{N}$ reaches the value of critic density just in an antecedent time to that of the recombination. Taking in consideration the dark matter existing 
inside Universe, we [1] build into $U_{H}$ a universe lattice of base $U_{d}$. We utilize the intersection-lattice between $U_{d}$ and a Baryonic universe-lattice $U_{B}$ (you see $\left(U_{d} \cap U_{B}\right)$ ) for build the actual universe-lattice of dark mass $U_{D}$ because we conjecture that the dark matter has a baryonic nature but interacting only gravitationally with the ordinary matter. We then calculate into $U_{H}$ the density of dark mass and we find a value next to one of critic density, so answering to question of missing mass.

In conclusion, the existence of a massive base camp $\left(\underline{\Xi}_{U}\right)$ leads us to think that also the dark mass can be related to this field. These affirmations tell that the dark mass is made by a massive scalar field of base $\left(\underline{\underline{\Xi}}_{D}\right)$, with a various spectrum of frequencies still to say (recall $\left[\underline{\boldsymbol{\Xi}}_{U} \Leftrightarrow\left(\underline{\boldsymbol{\Xi}}_{G}\right) \Leftrightarrow \underline{\boldsymbol{\Xi}}_{D}\right]$ ). We could then assume that the scalar field $\left(\underline{\Xi}_{U}\right)$ contains also the scalar field $\left(\underline{\underline{\Xi}}_{D}\right)$ associated with the dark mass detected in astronomical observations: $\left\{\left(\underline{\underline{\Xi}}_{U}\right) \supset\left[\left(\underline{\underline{\Xi}}_{D}\right), \underline{\boldsymbol{\Xi}}_{i}\right]\right\}$.

\subsection{The Evolution of "Universe at Lattice-Fields"}

It is evident in this paper that the idea of the universe structured in universes-lattice $\left(U_{i}\right)$ of base and crossing different evolving phases $\left(U_{k}^{*}\right)$ at variable metric doesn't oppose to the actual model of universe. Each phase reach the flat metric, you see [1] the Nucleosynthesis phase where wave propagates in fluid nuclear similar to that propagating in a fluid at flat metric, you see literature.

Rather it tries to resolve some problem list leading and it opens the way toward new horizons on the birth and evolution of the universe. By this new approach we have tried to face the fundamental problem of the expansion of the universe, explaining it as "creation" of space in the Universe Field generated in turn by mass creation in the universe. Not only but we prove to explain also the galactic acceleration through the ulterior mass-space creation inside a preexistent mass-space where it could create an "additional expansive pressure" (you see the following analogy: you imagine a crowded place of people where in any point of it there is the materialization of "one" person; it should happens that this last will create a pressure around it toward the others).

The weak point of this "new" cosmology should be the "mass creation". The first article [1] avoids the problem of the origin of the mass creation, by saying only that the universe observed by us could be built on some "lattice-fields" to "increasing mass" and to spatial expansion, because all data obtained by this model coincide with those observed astronomically. Instead, in present article, a relativistic cosmology with $(\Lambda)$, not added ad hoc, is derived using properly the equivalence between "creation of mass-space" and "expansion". In this article, we will hypothesize a state of "quantum vacuum" no built on the physical-mathematical aspect of the field constituted by a set of oscillators coupled in vacuum state; considering a quantum oscillator with semi-quanta and sub-oscillators (you see the idea of "IQuO" in Guido [2] [3]) we can think to new state of vacuum built by an aggregation of "empty" sub-oscillators (a sort of "sub-quantum vacuum"); this state cannot be considered a state of field and so cannot be insert in the mass-energy tensor of 
the Universe. The matter (antimatter) could be originated by energy flowing from the "sub-quantum vacuum" to fields which component the universe. These aspects can be inserted in the equation cosmologic without $(\Lambda)$ and to derive the complete cosmologic equation with $(\Lambda)$.

Besides, our theory being built on the lattices of field at massive coupling is coherent with the EP and can solely express a relativistic cosmology. Correlating the mass to space this theory "completes" the GR: the mass acts on the space in "totus", because the mass curves the space and produces it. Then, we will have that at "more mass" there is "more space" (see the Compton wave length). We could think so to new equivalence principle: the equivalence mass $\Leftrightarrow$ space. If the universe is the physical system of all lattices of field and these are increasing in the mass-space (and so increasing in "volume"), then universe comes seen in expansion and in acceleration. If we talk about [mass creation $\Leftrightarrow$ space creation] it is evident to propose the conjecture of an equivalence principle between mass and space. Nevertheless, the mass-space equivalence introduces a universe at variable metric, that, it clarifies some cosmological problems list not still resolved:

- The problem of Universe expansion problem described only mathematically and not physically by scale parameter $a(t)$ associated to galactic distances.

- The Dark Mass problem.

- The unconvincing hypothesis that the universe became at flat metric during the inflation phase (change of metric in a "isolated" universe).

- Expansive acceleration whose cause is a "mysterious" dark energy. Instead, ours new explanatory theses are remarkable:

- The physical explanation of the expansion as the effect of creating "space" and no through "dark energy".

- The creation of mass implies the creation of space or Space-Mass equivalence.

- The calculus [1] of time age in the phase $U_{H}$.

- The calculus [1] of density.

- The variable metric [1] caused by increasing mass.

- Possible mass value [1] of the dark matter and its density.

This cosmologic model with mass increasing describes so a universe in which in actual phase (Hydrogen phase $U_{H}$ ) is initially developed with variable open metric, it is reaching the flat phase after once of 14.82 billions of years from the Big Bang and then it should pass to a metrics closed with prevalence of the gravitational force; this last will determine a deceleration of the expansion until the stop for after begin a phase of contraction. So it is admitted the possibility that to end of this phase of contraction there will is a "Big Crunch". Nothing prohibits then another new Big Bang and therefore a new universe: we point out this possible universe as a "Cyclical Universe".

\section{Expanding Universe with Variable Metric}

\subsection{The Cosmologic Model of Einstein-Friedmann}

The solution for stationary universe formulated by Einstein gushed from a di- 
lemma: to introduce an opposing force to gravity without “... any justification physics" [4] or introducing “... ad hoc" an "additional” term $\left(\Lambda g_{i k}\right)$ to the metric "... permissible by relativity" which is not altering the covariance of the equations $\left[\left(R_{i k}-(1 / 2) g_{i k} R\right)+\Lambda g_{i k}+\chi T_{i k}=0\right]$ and compatible with a universe of “... finite" mass density. Nevertheless it could avoid ("mysteriously") the gravitational collapse because it inserts into Universe a "negative pressure...".

You understand here why Einstein called this (after the Hubble's discovery) an error: a position "ambiguous" to be deleted.

When Friedmann find solutions “... satisfactory”, Einstein stopped and did not investigated further about an issue which nevertheless is to contours still not “... sufficiently clarified". So Einstein thought that there was still something to say about the "space-time field of the Universe": like saying that the theory of gravitation is still "incomplete", seen it as a theory of space-time.

Believing that mass distorts space and come to a stop there, without trying to find a "co-natural" reciprocity between them, it can be a restrictive behavior which threatens to stop the speculation.

Reviewing so, briefly, what Einstein wrote on the solution of Friedmann to clarify the "incompleteness" of their solution is a must. In a note (see [4]), Einstein declared that in 1922 Friedmann proved [5] (before the discovery of Hubble) that the collapse of a Universe with "... finite density" can be avoided without the need to "... extend ad hoc the field equations" (i.e. without add $\left(\Lambda g_{i k}\right)$ ) if the distance between any two points (galaxies) is parameterized by introducing a calibration factor of space: $a(t)$. It is so obtained a cosmologic theory where is:

1) The RG equation states the connection between $S$-T geometry (Einstein tensor $\mathrm{G}$ ) and the mass-energy of the Universe (Tensor $T$ ): $G \Leftrightarrow T$.

2) The homogeneity and isotropy express the equivalence between cosmologic principle and the one the relativity.

3) The homogeneity and isotropy provides that the S-T lattice of Universe "fluid" can be expressed by a metric (RW) "parameterized", by expansion parameter $[a(t)]$, and by the curve parameter $(k)$ describing the three possible geometrical Space-Time of $U$.

4) The Bianchi's identity imposes a universe without "torsion": $D_{k}\left(G_{i}^{k}\right)=0$.

5) The U universe is "everything that is" and then total mass-energy is conserved if in Universe "fluid" there are no sources or wells: $D_{k}\left(T_{i}^{k}\right)=0$.

Therefore, that which avoids the gravitational collapse seems to be simply the introduction of the scale factor, perfectly compatible with the principle of relativity: a different calibration of distance (translation of "gauge" global) does not alter the laws of physics.

However, we note that despite the "perfect equivalence" of two principles, the introduction of $a(t)$ would be "ad hoc" if we do not ask what can determine the "scaling" of the universe which has as consequence the expansion (contraction).

Although the theoretical framework is coherent, there is not any hypothesis on the cause of these phenomena. 
In fact, the "incompleteness" of cosmology theoretical building of Einstein-Friedmann (EF) became evident in front of the discovery of the acceleration galactic: to explain this, immediately the cosmologists have resorted the "error" of Einstein.

In conclusion, in our opinion, since there is no explanation of galactic acceleration in the E-F theory, one must admit that their theory is incomplete. Of all that Einstein has had the suspect: “... issues... no sufficiently clarified".

However, it would still be "ad hoc" entering the parameter $(\Lambda)$, as well as the existence of the mysterious "dark energy" associated with it, if you do not look for the "source" of the same expansion; that is an inconsistency: to seek the source of acceleration of the expansion without seeking the source of the expansion itself. So, for completeness and consistency we must seek the source of the expansion.

Rethink $(\Lambda)$ no bump into objections of the same Einstein, it implies to think that the additional term in the "metric" should express an "action" of the space on himself which can "change" the his same metric by passing from closed at "open".

This "action" cannot be a "real" force (remember that the gravitational force is a false strength at light of equivalence principle), as well as its source cannot be an "agent". But a source the amending the metrics of space, as we know already, is the mass, therefore it is questionable whether it can become the source acting on the space by increasing its size, as if it "would create" space or as if it "would add" an ulterior additional space. We are so in front of the possibility of extending our way of seeing the relation [Mass $\Leftrightarrow$ Space] that would allow us to explain both the expansion that acceleration galactic.

\subsection{The Universe at Variable Metric}

So, we have shown that the origin of the universe expansion is to be found in a sort of" creation of space" in the (志 $)$-field which is built by the set of all the $\underline{\Xi}_{\dot{r}}$ Then at space creation in $\left(U_{S}\right)$, portion of $U$, we associate an "incoming" flow of space: $\Phi_{s}$

Seeing the expansion as space increasing it is equivalent to reaffirm that also the expansion is the expression of a particular property of the space (indicated with $\left.\underline{\boldsymbol{P}}_{\exp }\right)$. Instead we assign to gravity, which is understood as curvature of Space-Time, the property denoted with $\underline{P}_{\mathbf{g r v}}$. This distinction would deny that gravity (property $\underline{\boldsymbol{P}}_{\mathrm{grv}}$ ) can influence the expansion (property $\underline{\boldsymbol{P}}_{\exp }$ ) and vice versa. Furthermore, the experimental data seem to admit a $\left(V_{\text {rec }}^{*}\right)$ greater than that of an ordinary expansion, that is $\left[\left(V_{\text {rec }}^{*}\right)>\left(V_{\text {rec }}\right)\right]$ (see the Supernovae cosmology project [7]).

In particular, it is as if there was an "extra speed" ( $\left.V_{\text {ext }}\right)$ obtained by an acceleration opposite $\left(\boldsymbol{a}_{\exp }\right)$ to that of gravity $(g$ ) (as from the Supernovae cosmology project). In standard cosmology the $\left(V_{\text {ext }}\right)$ is consequence of a repulsive action generated by "dark energy" or by the energy of quantum empty. We sustain instead of explaining the $\left(\boldsymbol{a}_{\exp }\right)$ acceleration of the galaxies also through an increasing space.

Besides, note that if we associate to nascent masses an accretion of space or 
"space flow" in the universe then it is also evident to associate a "mass flow" to these neo-massive particles (you see the Compton wavelength $t_{0}=\hbar / m c$ ). We denote by "flow of Massa-Space" equivalence between the two flows. If the expansion is consequence of the flow space then it is evident that the corresponding mass flow has for consequence a "increase mass" of the Universe which produces a variable mass density, $\rho(t)$. Thus we will talk about a universe with Space-Mass in increasing and therefore with "variable metric".

This possibility, never incorporated into any cosmological model, was also highlighted by Einstein. In the presentation of the Friedmann model, Einstein [4] indeed he adds: “... There is no longer the assumption that the average density of matter is maintained over time... and it abandons the hypothesis that the expression of the metric field is independent of the time".

This implies the transition from the tensor $T_{i k}(\mathrm{EF})$-model to a tensor $\left(T_{i k}\right)^{*}$ to mass density variable (component $T_{00}^{*}$ ). So the Mass-Space equivalence requires to us that into universe a flow of Mass-Space is the cause of its expansion and acceleration as also of its variable density.

It remains to identify the "physics" source of this flow "entrant" in the universe which adds Massa-Space. We are so again, you see Einstein, front of a crucial dilemma to explain the current status of the universe or in explaining the origin of the "exceptional" phenomena such as expansion, its acceleration and the presence of dark matter.

These "exceptional" phenomena require a search of their origin which cannot be found between the known physical realities but, because of the incompleteness of the EF theory, in a new "logos" of physics. This search of the "source" pushes us to make new hypotheses that cannot be considered as "ad hoc" because it introduces new physical "subjects" which be lacking in the previous theory of EF, giving to it completeness and clearness (see Einstein).

\subsection{The Pressure of Gravitational Fall}

An astonishing feature is that the three solutions of Friedmann can be also derived in "Newton" contest, with the addition, in truth, of some "relativistic aspect" and of some "geometric" feature.

In a Newtonian context [5] it is possible to derive the acceleration of gravitational fall of the galaxies in comparison to ours. Thank to this acceleration we find the pressure of galactic gas that is present into Friedmann's solutions. In fact, in Newton it is

$$
F_{G}=G \frac{M_{U} m_{g a l}}{R^{2}}
$$

by $F_{G}=m_{\text {gal }} g_{\text {gal }}$ the galactic acceleration is

$$
g_{\text {gal }}=\frac{F_{G}}{m_{\text {gal }}}=G \frac{M_{U}}{R^{2}}=\left(\frac{G}{R^{2}}\right)\left(\frac{4 \pi}{3}\right)(\rho) R^{3}=\left(\frac{4 \pi}{3}\right)(G \rho R)
$$

or 


$$
g_{\text {gal }}(t)=\frac{4 \pi}{3} G \rho_{U}(t) R_{U}(t) .
$$

In a universe in expansion

$$
\left\{\begin{array}{c}
g_{\text {gal }}(t)=\left(\frac{4 \pi}{3}\right)\left(G \rho_{U}(t) R_{U}(t)\right) \\
\left(\frac{\mathrm{d}^{2} R(t)}{\mathrm{d} t^{2}}\right)=-g_{\text {gal }}
\end{array}\right\} \Rightarrow\left\{\left(\frac{\mathrm{d}^{2} R(t)}{\mathrm{d} t^{2}}\right)=-\left(\frac{4 \pi}{3}\right)\left(G \rho_{U}(t) R_{U}(t)\right)\right\}
$$

In a universe with matter in movement (galactic gas) and radiation (radiation gas) we have to add a general pressure: the "external" gas of radiations and galaxies acts with a pressure on a more inside stratum $\rightarrow P=F_{G} / S_{\text {sferica. }}$. Therefore the inferior stratum (you see Figure 7) will be submitted to an "external" force (always of gravitational origin) which a surplus of gravitational acceleration will engrave to it.

We calculate $\left(g_{\text {gas }}\right)$ :

$$
\begin{aligned}
g_{\text {gas }} & =\left(\frac{F_{G} / M_{\text {gas }}}{S_{\text {sferica }}}\right) S_{\text {sferica }}=\left(\frac{P}{M_{\text {gas }}}\right) S_{\text {sferica }} \\
& =P\left(4 \pi R^{2}\right)\left(\frac{1}{M_{\text {gas }}}\right)=P\left(4 \pi R^{2}\right)\left(\frac{G m}{\langle U\rangle R}\right) .
\end{aligned}
$$

The potential energy is assigned to $(M)$ as $(m)$, it follows

$$
\begin{gathered}
\left\langle U_{M}\right\rangle=\left\langle U_{m}\right\rangle=\left\langle E_{\text {rel }}(m)\right\rangle \approx\left\langle E_{0}\right\rangle, \text { where }\left[E=K_{\text {rel }}+E_{0} \text {; con } K_{\text {rel }} \ll E_{0}\right]: \\
g_{\text {gas }}=P\left(4 \pi R^{2}\right)\left(\frac{G m}{\langle U\rangle R}\right)=P\left(4 \pi R^{2}\right)\left(\frac{G m}{\langle E\rangle R}\right) \\
=P\left(4 \pi R^{2}\right)\left(\frac{G m}{m c^{2} R}\right)=\left(\frac{4 \pi R G}{c^{2}}\right) P
\end{gathered}
$$

where $\left\langle E_{0}\right\rangle=m c^{2}$.

It follows that:

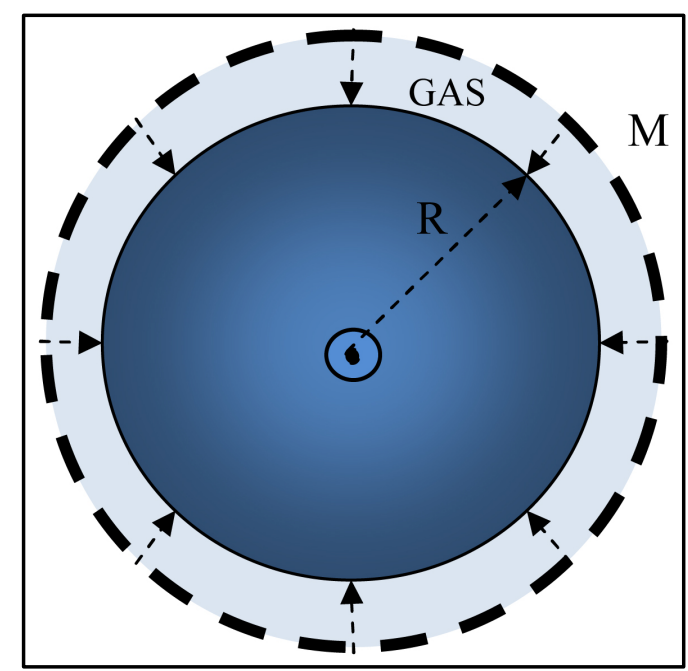

Figure 7. Gas pressure. 


$$
\begin{aligned}
g_{\text {tot }} & =\left(g_{\text {grav }}+g_{\text {gas }}\right)=\left[-\left(\frac{4 \pi G R}{3} \rho\right)-\left(\frac{4 \pi G R}{c^{2}} P\right)\right] \\
& =-\left(\frac{4 \pi G R}{3}\right)\left(\rho+\frac{3 P}{c^{2}}\right) .
\end{aligned}
$$

As we had already said this pressure increases the gravitational deceleration of a universe in expansion. Equally to $P_{\text {gas }}$ we can say that the mass-space flow can produce an additional pressure on the galactic matter.

\subsection{Pressure of Space-Mass Increasing}

In a universe with variable metric the $a_{\exp }$ could be related to additional pressure resulting from increasing space. Remember us that the Hubble's law, describing the Universe expansion, is the manifestation of the increasing space which happens for splitting of every IQuO of connecting (spatial cell) in a chain of $\Xi$. If it is introduced a spatial cell in a chain of IQuO of $\Xi$ then we can also think that this introduction will cause an "additional pressure" on the already existing space cell (you see Figure 8). By analogy we can think that in a place crowded with people suddenly a person it is materialized: it is clear that this will push the people around it, and that the pressure to edges boards is very little respect the one central.

Note in par. 4.3 we already demonstrated just that also in Newtonian way it can be see that the pressure of the galactic gaseous material increases the acceleration of gravity, thus agreeing with the relativistic treatment where the other diagonal components of tensor $T$ (or $T_{11} T_{11}, T_{22} T_{22}$ and $T_{33} T_{33}$ ) are the pressure and this (positive pressure) behaves like a mass and causes an attraction (see the Equation 4.6). Instead, the "additional" pressure is caused by the splitting of the space cells connected to IQuO [2] and this is different to galactic gas pressure expressed into tensor T of GR. The additional pressure is demonstrated be the origin of galactic acceleration which in literature instead is assigned to unknown dark energy; in this paper, on the contrary, the additional pressure is assigned to increasing mass-space that originates from a source (denoted by $\Theta$ ) with fieldless structure, named of "not field", and thus expressed not by tensor T (see more

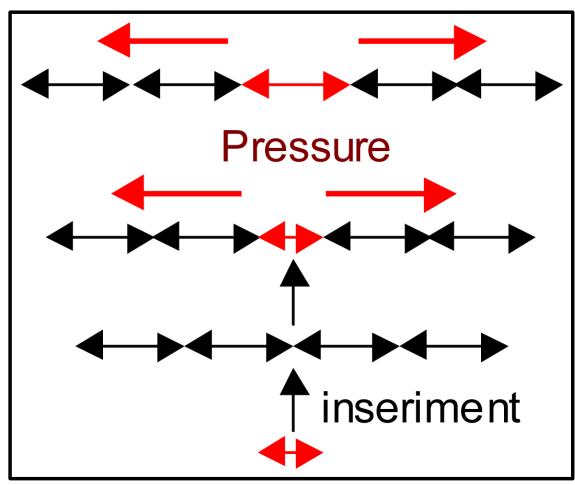

Figure 8. "Additional” pressure caused by the splitting of the space cells. 
forward paragraph 4.5) since this express all fields of the universe. In this way we add to the extended theories of gravity a new element of a possible solution to problem of dark energy.

Let's go to calculating the push and then the pressure of increase of space-mass.

Seeing that any model of expanding universe describes the galaxies placed on 4-dim surface, we consider a portion of this surface. If an IQuO-cell $(\lambda)$ emerges in a point of this surface, a push is produced all around the IQuO-cell of the chains of "surface"; it is established so a spherical cap with area ( $S=4 \pi R_{i}^{2}$ ), where $R_{i}$ is the radius of the surface corresponding to the length Compton associated with the cell-IQuO of the $(\Xi)$-field, step of the lattice defined on the surface. We will then have that $\left(S=4 \pi \lambda^{2}\right)$. The push will be given by $[F=\Delta p / \Delta t]$; it follows:

$$
\left\{p=\left(\frac{\hbar}{\lambda}\right), F=\left(\frac{p}{\tau}\right), c=\left(\frac{\lambda}{\tau}\right)\right\} \Rightarrow\left[F=\left(\frac{p}{\tau}\right)=\left(\frac{\hbar}{\lambda}\right)\left(\frac{c}{\lambda}\right)=\left(\frac{\hbar c}{\lambda^{2}}\right)\right] \text {. }
$$

The additional pressure or pressure of space increasing $\left(P_{\text {incr }}\right)$ will be:

$$
P_{\text {incr }}=\left(\frac{F}{S_{s f}}\right)=\left[\left(\frac{\hbar c}{\hbar^{2}}\right)\left(\frac{1}{4 \pi \pi^{2}}\right)\right]=\left(\frac{\hbar c}{4 \pi \hbar^{4}}\right) .
$$

Note that if we talk about pressure then we must talk about quanta: space-flow "implicates" quanta-flow. This will be for us that one which is said "dark energy".

The $\left(p_{\text {incr }}\right)$ pressure of "quanta" is similar to a gas pressure $\rightarrow\left[\left(p_{\text {incr }}\right) \equiv\left(p_{\text {gas }}\right)\right]$ with consequent acceleration of galactic gas $\left[g_{\text {gas }} \equiv g_{\text {incr }}\right]$; looking at Equation (4.7) also it follows that:

$$
P_{\text {incr }}=g_{\text {incr }}\left(\frac{c^{2}}{4 \pi R G}\right) .
$$

Here is $\lambda \rightarrow R$, so considering the pressure to "edges" of any spherical cap. We specify that the $P_{\text {incr }}$ although it is a local pressure, will be present in the entire universe. This aspect is typical of a "hydrostatic pressure", in fact you see the aspect of perfect fluid assigned to the universe. Some notes:

A first note is the one that the $P_{\text {incr }}$ is "inside" $U$ or in $\left(\underline{\Xi}_{U}\right)$.

A second note: if the universe is closed (on itself) then the accretion pressure would be nil. It follows that the accretion pressure is only present in an "open" universe with negative curvature or nil (flat universe).

At the beginning of the birth of the lattice massive had to be:

$$
P_{\text {incr }}=g_{\text {incr }}\left(\frac{c^{2}}{4 \pi \hbar G}\right) .
$$

The accretion pressure can be seen as a negative pressure which is opposite to that gravitational.

Putting $\left[\left(p_{\text {acr }}\right) \equiv\left(p_{\text {gas }}\right)\right]$ we find the expression of $\left(g_{\text {incr }}\right)$ which now can be seen as an acceleration of anti-gravity $\left(g_{\text {anti-g }}\right)$. Therefore we will have $\left[\left(g_{\text {exp }}\right) \equiv\left(g_{\text {incr }}\right) \equiv\right.$ $\left.\left(g_{\text {anti-g }}\right)\right]$; then we will have that: 


$$
g_{\text {incr }} \equiv\left(g_{\text {anti-g }}\right)=\left(\frac{F_{\text {gas }}}{m}\right)=\left[\left(\frac{\hbar c}{\hbar^{2}}\right)\left(\frac{1}{m}\right)\right]=\left[\left(\frac{\hbar c}{\lambda^{2}}\right)\left(\frac{\lambda c}{\hbar}\right)\right]=\left(\frac{c^{2}}{\hbar}\right)
$$

Replacing we get:

$$
P_{\text {incr }}=g_{\text {anti-g }}\left(\frac{c^{2}}{4 \pi \lambda G}\right)=\left(\frac{c^{2}}{\lambda}\right)\left(\frac{c^{2}}{4 \pi \hbar G}\right)=\left(\frac{c^{4}}{4 \pi \hbar^{2} G}\right)=\left(\frac{2}{\lambda^{2} \chi}\right) .
$$

$P_{\text {incr }}$ is the pressure on unity of IQuO-cell. Here $\left[\chi=\left(8 \pi G / c^{4}\right)\right]$ is the [4] same constant of the equation of general relativity $\left[G_{i j}=\chi T_{i j}\right]$.

Referring to the "edges" of cap with radius $R$ and remembering the "hydrostatic pressure" in each point of Universe, we should have, for $\lambda \rightarrow R$ :

$$
P_{\text {incr }}=\left(\frac{2}{R^{2} \chi}\right) \text {. }
$$

The accretion pressure can so well describe a constant negative pressure $\left(P_{\Lambda}\right)$ in whole universe.

In the "edges" $\left(g_{\text {anti-g }}\right)$ can be defined as additional acceleration of type "centrifugal" ( $\left.a_{f}\right)$ given by $\left[\left(\left(V_{\text {exp }}\right)^{2} / \mathrm{R}\right]\right.$ because the "force" from pressure of space-mass accretion pushes to "outwards". It follows $\left[a_{f}=g_{\exp }\right]$.

Having found that $\left[g_{\exp }=a_{f}=\left(v_{f}\right)^{2} / R\right]$; it follows ( for $v_{f}=c$ )

$$
g_{\text {exp }} \equiv g_{\text {incr }} \equiv\left(g_{\text {anti-g }}\right)=\left(\frac{c^{2}}{R}\right) \text {. }
$$

The $\left(g_{\text {exp }}\right)$ as $\left(g_{\text {gas }}\right)$ will be:

$$
g_{\exp }=\left(\frac{4 \pi G R P_{\exp }}{c^{2}}\right) .
$$

The accretion pressure can so well describe the negative pressure that in the cosmology is associated with the cosmological constant in whole universe, indicated with $\Lambda_{s}$.

$$
P_{\Lambda}=\left(\frac{\Lambda_{s} c^{4}}{8 \pi G}\right)=\left(\frac{\Lambda_{s}}{\chi}\right)
$$

We compare the $\left(P_{\Lambda}\right)$ pressure cosmological with $g_{\exp }$

$$
\left\{\begin{array}{l}
g_{\exp }=\left(\frac{4 \pi G R P_{\exp }}{c^{2}}\right) \\
P_{\text {esp }}=P_{\Lambda}=\left(\frac{\Lambda_{s} c^{4}}{8 \pi G}\right)
\end{array}\right\} \Rightarrow\left\{g_{\exp }=\left(\frac{4 \pi G R}{c^{2}}\right)\left(\frac{\Lambda_{s} c^{4}}{8 \pi G}\right)\right\} \Rightarrow\left\{g_{\exp }=\left(\frac{\Lambda_{s} R c^{2}}{2}\right)\right\} .
$$

Comparing $\left(g_{\exp }\right)$ with $\left(g_{\text {incr }}\right)$ we will have

$$
\left\{\begin{array}{c}
g_{\exp }=\left(\frac{R \Lambda_{s} c^{2}}{2}\right) \\
g_{\exp }=\left(\frac{c^{2}}{R}\right)
\end{array}\right\} \Rightarrow\left\{\left(\frac{c^{2}}{R}\right)=\left(\frac{R \Lambda_{s} c^{2}}{2}\right)\right\} \Rightarrow\left\{\Lambda_{s}=\frac{2}{R^{2}}\right\} .
$$

If we put inside $[R=c \tau]$, then it is $\left[\Lambda_{\tau}=\Lambda_{s} c^{2}\right]$ with $\Lambda_{\tau}$ the cosmological con- 
stant of actual cosmology standard.

A comparison shows that $\left[\Lambda \Leftrightarrow \lambda\left(U_{i}\right)\right]$ or the cosmological constant refers to the pitch of the lattice in accretion.

Instead we recall that in cosmology therefore the pressure associated with the cosmological constant is:

$$
P_{\Lambda}=\left(\frac{\Lambda_{s} c^{4}}{8 \pi G}\right)=\left(\frac{\Lambda_{s}}{\chi}\right)
$$

Having (you see Equation (4.13)) so that $\left(P_{\Lambda}\right) \Leftrightarrow\left(P_{\text {exp }}\right)$.

We will have the beginnings (when a lattice-universe is made up of a few IQuO coupled) that:

$$
\left\{\begin{array}{c}
\Lambda=\frac{2}{\lambda_{p l}^{2}} \\
\lambda_{p l}=1.616252 \times 10^{-35} \mathrm{~m}
\end{array}\right\} \Rightarrow\left[\Lambda=0.765617 \times 10^{70}\left(1 / \mathrm{m}^{2}\right)\right] .
$$

Calculating the pressure

$$
P_{\text {esp }}=\left(\frac{\Lambda c^{4}}{8 \pi G}\right)=\left(\frac{2.99792458^{4} \times 10^{32} \times 0.765617 \times 10^{70}}{(8 \pi) \times 6.67259 \times 10^{-11}}\right) \mathrm{Pa}=3.6877 \times 10^{112} \mathrm{~Pa} \text {. }
$$

In the current universe we have instead $R \rightarrow R_{U}$ [where $R_{U}$ is calculated by Universe time of Planck's telescope]:

$$
\left\{\begin{array}{c}
\Lambda=\frac{2}{R_{U}^{2}} \\
R_{U}=13.07 \times 10^{25} \mathrm{~m}
\end{array}\right\} \Rightarrow\left[\Lambda=1.17 \times 10^{-52} \mathrm{~m}^{-2}\right] .
$$

This result is very close ([7]) to the experimentally calculated: $\Lambda \approx 10^{-52} \mathrm{~m}^{-2}$.

So we can specify that the "Dark Energy" (noted in literature) is identified in the additional pressure caused by space flow or by "quanta" flow entering in the Universe or in the field $\left(\underline{\Xi}_{U}\right)$ which coming be an expansive field.

\subsection{The Source of the Pressure from Mass-Space Flow}

Now the dilemma is the one of find the source (denoted by $\Theta$ ) of the incoming flow of Mass-Space in the Universe Field $U$, which determine the additional pressure accelerating the galaxies. Because $U$ is understood as all of the one that we can empirically know, then $(\Theta)$, being source of empiric phenomenon, cannot be outside $U$. Having talk about Universe as a "Universe-Field" (you see $\left.\left(\Xi_{U}\right) \equiv\left(\Xi_{p} \Xi_{j}\right)\right)$, we can have two possibilities on the nature of system $(\Theta)$ :

1) $(\Theta)$ is new form of particle-field: this possibility is said "field solution" (you see the hypothesis of the "dark energy" in literature).

2) $(\Theta)$ is new "status" of the "energy" which not involves the field form: this possibility we said be "not-field solution".

We take in consideration the second original possibility for clear motives: the first is that the form of dark energy is not known.

We remember that the field is made by a system of coupled oscillators "elasti- 
cally"; from here the particles described by wave equations. Thus the status of system $(\Theta)$ could be a physical state made by a set of no-coupled quantum oscillators (you see [2] the idea of IQuO). It is evident that we cannot talk of "interaction" between the two systems $U$ and $(\Theta)$ through an interacting field.

The second solution implicates to consider a "generalized universe" " $U^{\star}$ " composed by $(U, \Theta):\left[U^{\star}=U \oplus(\Theta)\right]$.

To understand the equivalence between $\Theta$-system and set of quantum oscillators uncoupled, we have resort to a new representation of quantum oscillator which can admit a vacuum state of uncoupled quantum oscillators. In [2] one represents a quantum oscillator composited by two sub-oscillators with semi-quanta (IQuO) (see Figure 9):

The quantum oscillator of field no longer will described by the pair of operators $\left(a, a^{+}\right)$but by two pair of operators $\left[\left(a_{e l}, a_{e l}^{+}\right),\left(a_{i n}, a_{i n}^{+}\right)\right]$where the absorptive component becomes the inertial component. This double structure of the operators $\left(a, a^{+}\right)$splits the energy quanta in the quantum oscillator, giving:

$$
\begin{aligned}
{\left[H_{(n)}\right] } & =\left[U_{(n)}+K_{(n)}\right]=\left[\left(U_{(n)}\right)_{e l}+\left(K_{(n)}\right)\right] \\
& =\left[(2 n+1)\left(\frac{1}{4} \hbar \omega\right)_{e l}+(2 n+1)\left(\frac{1}{4} \hbar \omega\right)_{i n}\right] .
\end{aligned}
$$

A structure with two sub-oscillators involves that when the energy quantum is in one of two sub-oscillator the other sub-oscillator cannot be empty of energy but having value of $(\varepsilon=1 / 2 h v)$ defined "semi-quantum" (remember [ $\varepsilon_{(n=1)}=$ $(1+1 / 2) h v)])$. A structure with two components (elastic and "absorbing" or inertial) and two sub-oscillators would say that the "quantum" is composed by two $(\varepsilon=1 / 2 h v)$ semi-quantum. Then we conjecture energy values of $(\varepsilon=1 / 4 h v)$, indicated as "empty semi-quantum" and symbol (o), and another [ $\varepsilon=(1 / 2 h v)]$, indicated as "full semi-quantum" and symbol (•). The vacuum state of IQuO will be represented by one only sub-oscillator which must be in isolated state with energy:

$$
\left[H_{(0)}\right]=\left[\left(U_{(0)}\right)_{e l}+\left(K_{(0)}\right)_{\text {in }}\right]=\left[\left(\frac{1}{4} \hbar \omega\right)_{e l}+\left(\frac{1}{4} \hbar \omega\right)_{\text {in }}\right] .
$$

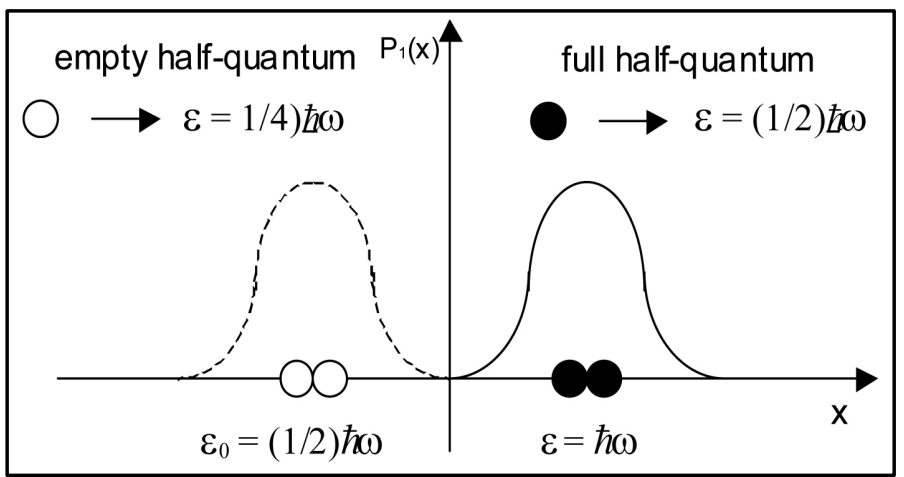

Figure 9. Probability function in quantum oscillator with $(\mathrm{n}=1)$. 
The vacuum state of an "isolated" IQuO will be graphically represented in Figure 10.

Then we can define a physical system composed by a set of sub-oscillators with one full half-quantum: pair $(\mathbf{o}, \bullet)$; this system has energy but has not any structure of field. Therefore, the vacuum state of an IQuO uncoupled is different by that of the "classic" quantum oscillator and allows the existence of an "isolated" oscillator. The IQuO-representation could so allow the existence of physical system composed only by set sub-oscillators in vacuum state and uncoupled $(\Theta)$ or of "not-field".

The present paper is original in contest of the extended theories of gravity (see Christian Corda [8]) for its new idea about vacuum quantum of "not-field"; this do not can be treated to same way of vacuum of quantum theory of field because its properties are different and are relative to a new state of quantum oscillator not coupled which specifically defines the state of not field.

To better understand the state of not field, we recall the Higgs mechanism where are defined some fields $(\phi)$ whose energy potential $V(\phi)$ is different from zero even when the field assumes a null value. This means that these fields admit a non-zero energy value with null field. The representative oscillators of these fields are always coupled between them even when they are in the vacuum state [ $V=$ 0 ] and, for literature, in the null state of field [ $\phi=0$ ]. If we introduce the idea of $\mathrm{IQuO}$ (see Guido [2]) where a quantum oscillator in vacuum state $(n=0)$ consists of a single sub-oscillator (while for $n=1$ there are two sub-oscillators, as can be seen in the wave function of the quantum oscillator), then we can admit quantum oscillators in decoupled vacuum state which don't express any field. This involves the possibility of having a new form of quantum vacuum that we will define of non-field by the fact that the oscillators are uncoupled. The physical possibility of having quantum oscillators in the uncoupled state or of not-field pushes us to admit a physical state $(\Theta)$ into universe that don't contributes to contents in mass-energy ( $T$ tensor) because in "quiescent" state and because do not contains energetic fields ( massive and not).

Therefore, the evolution of the universe would be the result of a continuous exchange of a semi-quanta flow of energy between these two systems $(U, \Theta)$.

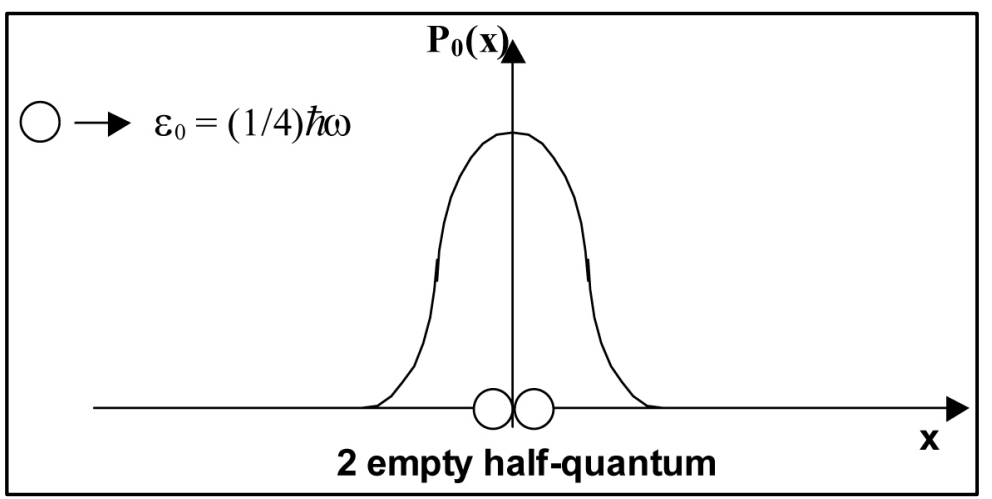

Figure 10. Probability function in sub-quantum oscillator with $(\mathrm{n}=0)$. 
There are two different possibilities:

- The pouring of semi-quanta from $(\Theta)$ to $U$ happens "wherever" in $U$ (this because $(\Theta)$ is "underground" in $\left(\underline{\Xi}_{U}\right)$ ) for obtain a homogeny and isotropic expansion.

- It could exist some specific "gates" placed in way homogenous "wherever" in $U$, like the galactic black holes.

Remember that on $\left(\underline{\Xi}_{U}\right)$ there are structured two form of mater: the dark mass $\left(\underline{\Xi}_{D}\right)$, which is a base field of mass no-radiant, and the ordinary mass $\left(\underline{\Xi}_{O}\right)$, radiant structure forms (particles of standard model). Then we have admit that a part of flow of energy (quanta) going from $(\Theta)$ to Universe $(U)$ origins the dark matter [1].

But, in order to "mass-space" equivalence, these asserts lead us to speak of the mass-space flow coming from $(\Theta)$ gives rise also to mass-energy dark [ $\underline{\Xi}_{\mathrm{Dm}}$, $\left.\Xi_{\mathrm{De}}\right]$, where $\left(\underline{\Xi}_{\mathrm{De}}\right)$ is the "mass-space" field causing the galactic acceleration (or dark energy). This is a very important news and original.

The equivalence mass-space pushes us to admit after that there is an equivalence between dark mass flow and space flow (pressure of dark energy) and this should determines that $\left(\Omega_{\mathrm{dm}} / \Omega_{\mathrm{de}} \approx 1\right)$.

Going forth we will understand $(\Theta)$ as a "perfect fluid" contained into a "inert underground container" into Universe which come out (as mass-space flow) and so gives rise to the mass-space, such as fields $\left[(\underline{\Xi}),(\underline{\underline{\Xi}})_{o}, \underline{\Xi}_{\mathrm{Dm}}, \underline{\Xi}_{\mathrm{De}}\right]$, giving rise to the space increase, which is observed experimentally as galactic expansion and additional pressure that accelerates galaxies.

Now we ask about the physical properties of $(\Theta)$. It is very important to note that the pressure of the space-mass flow arises within the universe $U$ or in the whole field $\Xi_{U}$. There is a pressure only when a cell of mass-space is originated in $\left(\Xi_{U}\right)$ or this is inserted into a pre-existing chain $\left(\underline{\underline{\Xi}}_{i}\right)$ of $\left(\Xi_{U}\right)$ through a mechanism not yet defined. An analogy can help us: in a missile, only after the internal combustion, the gas, during the expulsion from the reactor, applies a push on the missile. Thus, we have to admit a sort of dynamically "inert" container (as the one of missile), which supplies energy quanta for building quantum oscillators into base field $\left(\Xi_{U}\right)$ or even building "cells" of $(\underline{\Xi})$ mass-space into $\left(\underline{\Xi}_{U}\right)$ field. Seeing that whatever interaction is represented by an intermediary agent with a specific structure of field, we are forced to admit that an "inert" dynamically system, although having energy quanta, cannot have a structure of field. Thus it is intuitive to admit that only an elastic coupling between quanta on the "boundary surface" of the container allows of passing from the "inert" state to one of "field", causing the birth of field oscillators $\left(\Xi_{U}\right)$ or $\left(\Xi_{U}\right)$ : the rising of new cell of mass-space builds chains in ( $\left.\underline{\Xi}_{U}\right)$ (this is expansion) but only the inclusion of other new space-cell into chains determines subsequently the accelerating pressure. A precise statement: accelerating pressure is so present only between oscillators belonging to field of base $(\underline{\underline{\Xi}})$ which represent the reference frame "comovent". Therefore, the $(\Theta)$ cannot interact with matter of the universe. Be- 
sides we note that whole set of the quanta in "inert" state (into the container) is a physical system more fundamental of the fields $\left(\Xi_{U}\right)$ o di $\left(\Xi_{U}\right)$. Therefore the $(\Theta)$ system defines an "inert" state of "no-field", which somehow is "underground container" into U.

We remember also that the state of the quantum vacuum is still a state of field or physical system made of coupled quantum oscillators although in a state of "empty". Therefore the $(\Theta)$ system could define a new idea of "quantum vacuum": the one of quantum vacuum of no-field.

In summary the dark energy cannot longer be associated with the quantum vacuum of fields but it will be associated to mass-space flow which extracts energy from the inert "container" system $(\Theta)$, having a structure of no-field, and it induces an additional pressure on the matter giving origin to acceleration of the galaxies. Talking about Universe on the pressure of mass-space increasing it is equivalent to affirm that a flow (for “osmosis"?) of energy (quanta) goes from $(\Theta)$ to $\left(\Xi_{U}\right)$ or on $\left(\Xi_{U}\right)$ of the Universe $(U)$.

Remember that on $\left(\underline{\Xi}_{U}\right)$ there are structured two form of mater: the dark mass $\left(\Xi_{D}\right)$, which is a base mass no-radiant, and the ordinary mass $\left(\Xi_{O}\right)$, radiant structure forms (particles of standard model).

Then we have admit a flow of energy (quanta) going from $(\Theta)$ to Universe $(U)$ which gives origin also at dark matter.

If the Universe is as a Space-Time Field $\left(\Xi_{U}\right)$, the $(\Theta)$-system, being a no-field, is out of the space and time, even if it has energy in quantity to finish. This is because $(\Theta)$ is made up of no-coupled quantum oscillators in vacuum state and completely "free" like the atoms of a "perfect fluid" (so like one see actuality the Universe).

An important observation: being $\Xi_{U}$ a massive-lattice it follows that the Universe is also increasing in mass with the significant consequence to have a $\left(\rho_{U}\right)$ variable mass density [1].

So we can say that:

- A whatever mass flow (from preexisting mass-space) gives rise both the "dark mass" and the "ordinary mass", so inducing a variation of mass density of Universe (you see the increasing lattice-fields).

- A whatever space flow (from preexisting mass-space) gives rise the expansion of the universe.

- A space flow (mass-space) coming from $(\Theta)$ gives rise the additional pressure which accelerates the galaxies (in literature this is the "dark energy").

- A mass flow (mass-space) coming from $(\Theta)$ gives rise at the "dark mass" and the "ordinary mass".

Going forth we will understand $(\Theta)$ as a "perfect fluid" contained into a "underground" inert container into Universe that come out (as mass-flow space) and so give rise to the mass-space, such as fields $\left[(\Xi, \Xi)_{O}, \Xi_{D}\right]$, to give rise the space increase, which is observed experimentally as galactic expansion, and also to give rise the additional pressure that accelerates galaxies. 
We do not exclude the possibility that also creation of ordinary mass of base (leptons and quarks) gives origin at expansion because the mass flow is as ever "equivalent" at space-flow (the expansion).

Once that we have admit the existence of $\Theta$, it is necessary to understand "where" is happening to pour of quanta from $\Theta$ to $U$.

Having said that into universe a scalar field of base $\left(\underline{\Xi}_{U}\right)$ exists wherever, thus also $\Theta$ will be wherever (but in underground to $\left(\underline{\Xi}_{U}\right)$ ) and it gives origin indirectly in everybody at the processes of creation of mass (because it gives quanta to $\Xi$ and $\Xi$ by the mass-energy-space flow which increases $\left(\Xi_{U}\right)$ with consequently expansion and variable mass density) or directly (you see the dark mass and the additional pressure which gives acceleration at galaxies).

It is clear then that we can substitute the initial singularity of the Big Bang with the not-field $\Theta$. The cosmic time so has had origin when the quanta flow from $\Theta$ has had the start. Now there are two possibilities on the idea of "wherever": the decanting of quanta from $\Theta$ to $U$ happens "wherever" in $U$ (remember that $\Theta$ is underground in $\left.\left(\Xi_{U}\right)\right)$ or it happens crossing the some "doors" placed in way homogenous "wherever" in $U$, like can be the galactic black holes.

We believe that both two possibilities happening into $U$.

The first possibility is consistent with the law of Hubble drawn in a universe to mass-space increasing (expansion), where the splitting of the IQuO happens in everybody the points of the space (see par. 2.4)

As also (second possibility) the additional pressure is due to mass-space flow which has origin into Black Hole $(\mathrm{BH})$. This because at the center of any galaxy there may be a Black Hole [9], then we believe that a $(\mathrm{BH})$ could be a possible other "entrance" of the flow of quanta from $\Theta$ to $\left(\underline{\Xi}_{U}\right)$ which forms the field $\left(\Xi_{D}\right)$ : the BH can be seen like a "hydraulic" pump which extracts quanta from $\Theta$ and it puts them into $U$ in form of "dark mass". You see the following Figure 11.

The analogy is that of a submarine volcano erupting lava: this gives rise around itself rock on rock that will take it to emerge as a rocky island generating then

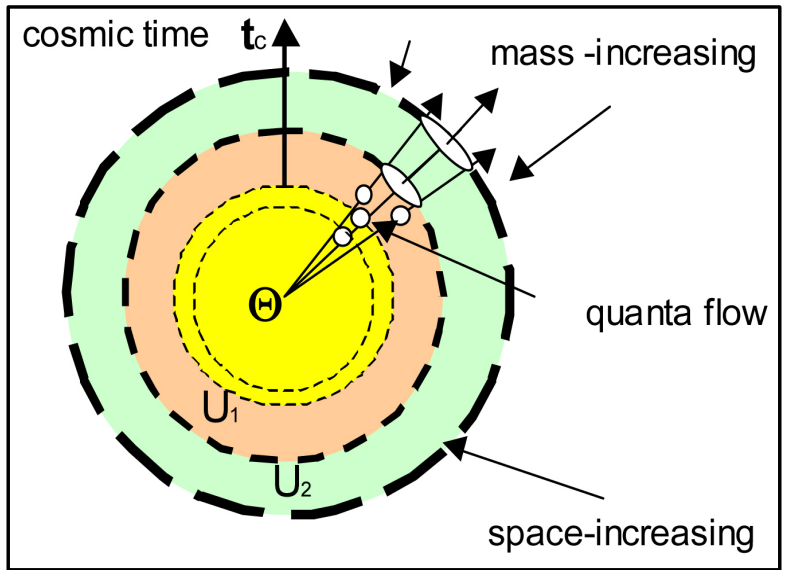

Figure 11. Two systems $\left(\Theta-\Xi_{U}\right)$. 
the earth itself.

Nevertheless we believe that the "dark matter" is correlated with "dark energy", seeing the first like mass flow coming out from galactic black holes while the second like space-flow which is accelerating the galaxies (you see the question of cosmic coincidence).

The dark matter could have so origin into quanta flow which flows from $\Theta$ to $\left(\underline{\Xi}_{U}\right)$ : all this builds so the field of dark matter $\left(\underline{\Xi}_{D}\right)$, which wraps any galaxy.

\section{The Cosmological Equation}

\subsection{Einstein Cosmological Equation}

The Einstein's cosmologic equation:

$$
\left\{\left[R_{i k}-\frac{1}{2} R g_{i k}\right]=-\chi T_{i k}\right\} \Leftrightarrow\left\{G_{i k}=-\chi T_{i k}\right\} .
$$

This equation describes a Universe with the following properties:

1) $\partial T_{i k}=0 \Leftrightarrow T_{i ; k}^{k}=0$.

2) The $(k)$ curvature constant is invariant in typology but no in value.

This because a surface in expansion doesn't change the curvature typology (open, plat or closed) but only the value, recalling that the curvature ray in an expansive universe goes toward zero value (plat metric). Besides, the galactic acceleration pushes the cosmologists to readmit the Einstein's constant $(\Lambda)$ into cosmologic equation as follows:

$$
G_{i k}=-\chi T_{i k}-\Lambda g_{i k} .
$$

For current cosmology the $(\Lambda)$ provides the $\left(P_{\text {exp }}\right)$ pressure accelerating the galaxies. The source of this pressure could be the dark energy drawn by quantum vacuum but this origins problem has infinites numerical values in physical variables. On the contrary, in this new approach the expansion and additional pressure are consequence of "mass-space increasing" in Universe. Another consequence is the possibility that the metric changes not only of value but also of type. If we admit the mass-space flow then the cosmologic equation takes into account the following particular conditions:

- The descriptive coordinates are formulated between $a(t)$ gauge parameter, consequent to space flow.

- Into the equation is present a tensor $\left[\left(\Lambda_{i k}\right)=\Lambda g_{i k}\right]$, in addition to Ricci's tensor $\left(R_{i k}\right)$, which expresses the metric variation for expansion and where the $(\Lambda)$ parameter describes the additional pressure consequent at space flow (additional acceleration of galaxies) .

- Into mass-energy tensor $\left(T_{i k}\right)$ the mass density can be variable because there is a meaningful incoming mass flow into $U$.

In this context it is not more admissible that $\left(\partial T_{i k}=0\right)$ and that the $G_{i k}$ metric tensor can express the space curvature as function of only mass-energy: we would admit that $G_{i k}$ is so function of accretion of space at the flowing of "cosmic" time. 


\subsection{Variational Principle in Cosmology}

We ask us how could be a cosmological equation with flows of mass and space. We start from a geometric aspect: the expansion of a surface induces variations to curvature value because the curvature ray is increasing and if $\left(R_{c} \rightarrow \infty\right)$ it follows that $k \rightarrow 0$. This is equivalent to argue that an expanding universe, both it be open metric $(k<0)$ or it be to closed metric $(k>0)$, it would tend to become a flat space. However the metric of a specific space (with $k$ values given) could change if the particular scale factor $a(t)$ is variant to pass of the time. This is possible in the theory of a universe with mass-space increasing, because there are the flows of space and mass which could change the value of the mass density $\left(\rho_{U}\right)$ and, therefore, the $(k)$ and thus $a(t)$. In this new cosmology the curvature parameter $(k)$ could vary from value $(k=-1)$ to $(k=+1)$, passing through $(k=$ $0)$.

We face the question in analytical terms considering the action function and the principle of Hamilton. It is possible draw the cosmologic equation by variational principle [10] applied to physical system mass-energy (with the $\mathfrak{E}_{m}$ Lagrangian and action $S_{m}$ ) and to gravitational field (with Lagrangian $\mathfrak{E}_{g}$ and action $S_{g}$ ).

We will have

$$
\left\{\begin{array}{c}
S_{m}=\int £_{m}\left(g_{i k}, \partial g_{i k}\right)(\sqrt{-g}) \mathrm{d} \Omega \\
S_{g}=\delta \int £_{g}\left(g_{i k}, \partial g_{i k}\right)(\sqrt{-g}) \mathrm{d} \Omega
\end{array}\right\} .
$$

Here $g_{i k}$ is metric tensor. The Hamilton's principle admits that:

$$
\left[\delta\left(S_{m}+S_{g}\right)=\delta S_{U}=0\right] .
$$

However, you cannot build a universe scalar containing only terms $(g, \Gamma)$ because the $\Gamma_{i k}^{j}$ coefficients can vanish in a point of the space and $(g)$ become Galilean coordinate system (or a local reference frames); we cannot use the $(R)$ Ricci scalar in $\mathfrak{E}_{g}$ because $(R)$ contains also second derivatives.

Yet we could use (you see Landau [10]) $(R)$, in linear form, as combination of two terms $[R \equiv G+W]$ or

$$
\begin{aligned}
& {\left[\int R(\sqrt{-g}) \mathrm{d} \Omega\right]=\left[\int G(\sqrt{-g}) \mathrm{d} \Omega\right]+\int\left(\frac{\partial\left(w^{l} \sqrt{-g}\right)}{\partial x^{l}}\right) \mathrm{d} \Omega} \\
& \{R\}=\{G\}+\{W\} \\
& =\left[\int G(\sqrt{-g}) \mathrm{d} \Omega\right]+\int(\operatorname{div} \underline{w}) \mathrm{d} \Omega \text {. }
\end{aligned}
$$

Here $\underline{w}=W^{\cdot} \cdot(-g)^{1 / 2}$. The term $(\mathrm{G})$ contains only $g_{\text {k }}$ and $\left(\partial g_{i k}\right)$ as first order derivatives, while the term $(W)$, the divergence $[\operatorname{div} \underline{w}]$, contains the second derivatives of $g_{i k}$ In this form the Ricci scalar could describe a gravitational field if in somehow the term $(W)$ disappears to $S_{g}$ variation. In fact this happens: recalling Stokes, the integral with the divergence becomes an integral flux $[\Phi(\underline{w})]$ but since the variation of the divergence (obtained varying the action $S_{g}$ ) contains variations of $g_{i k}\left(\right.$ or $\left.\delta g_{i k}\right)$ which, to integration extremes, are zero, it follows that the flow would be zero. In this way $\delta S_{g}$ coincides only with the variation of the first integral, the 
one that contains the $(G)$, and not with that of the second (which vanishes). The scalar $(G)$ is thus the sole survivor of the scalar $(R)$ with the form:

$$
\begin{array}{llc}
R=\left\{\left[g^{i k}\left(\Gamma_{i l}^{m} \Gamma_{k m}^{l}-\Gamma_{i k}^{l} \Gamma_{l m}^{m}\right)\right]\right. & \left.+g^{i k}\left[\frac{\partial \Gamma_{i k}^{l}}{\partial x^{l}}-\frac{\partial \Gamma_{i l}^{l}}{\partial x^{k}}\right]\right\} \\
\{R\} \equiv & \{G\} & \{W\} .
\end{array}
$$

If we have

$$
\sqrt{-g} R=\sqrt{-g}\left(g^{i k} R_{i k}\right)
$$

calculating that

$$
\sqrt{-g} g^{i k} \frac{\partial \Gamma_{i k}^{l}}{\partial x^{l}}=\frac{\partial}{\partial x^{l}}\left(\sqrt{-g} g^{i k} \Gamma_{i k}^{l}\right)-\Gamma_{i k}^{l} \frac{\partial}{\partial x^{l}}\left(\sqrt{-g} g^{i k}\right) .
$$

Substituting into (5.7) and neglecting the two derivatives total we find that the development of $(R)$ coincides with $G[R=G]$. In this way one solves the problem of find a $\mathfrak{E}_{g}$ which describes a gravitational field being in any point of the space. Not only, but field variations $\left(\delta g_{i k}\right)$ in the extremes of integration and the outward flux from $S$ surface of the vector $(w)$ are related issues. The $(W)$ term is again found in the variation of action $S_{g}$; in fact if we use the following relations:

$$
\left\{\begin{array}{c}
\sqrt{-g} R=\sqrt{-g}\left(g^{i k} R_{i k}\right) \\
\delta(\sqrt{-g})=\frac{-1}{2(\sqrt{-g})} \delta(-g)=\frac{-1}{2} \frac{(\sqrt{-g})}{g} g g_{i k} \delta g^{i k}=-\frac{1}{2}(\sqrt{-g}) g_{i k} \delta g^{i k}
\end{array}\right\}
$$

we get

$$
\begin{aligned}
\delta S_{g} & =\delta\left[\int R(\sqrt{-g} \mathrm{~d} \Omega)\right]=\delta\left[\int g^{i k} R_{i k}(\sqrt{-g} \mathrm{~d} \Omega)\right]=\delta\left[\int R_{i k}(\sqrt{-g}) g^{i k}(\mathrm{~d} \Omega)\right] \\
& =\left[\int R_{i k}\left(\sqrt{-g} \delta g^{i k}\right)+R_{i k}\left(g^{i k} \delta \sqrt{-g}\right)+g^{i k}(\sqrt{-g}) \delta R_{i k}\right] \mathrm{d} \Omega \\
& =\left[\int\left(R_{i k}-\frac{1}{2} g_{i k} R\right) \delta g^{i k}(\sqrt{-g}) \mathrm{d} \Omega\right]+\left[\int\left(g^{i k} \delta R_{i k}\right)(\sqrt{-g}) \mathrm{d} \Omega\right]
\end{aligned}
$$

where the $\left(R_{i k}\right)$ tensor is

$$
R_{i k}=\left\{\left[\left(\Gamma_{i k}^{l} \Gamma_{l m}^{m}-\Gamma_{i l}^{m} \Gamma_{k m}^{l}\right)\right]+\left[\frac{\partial \Gamma_{i k}^{l}}{\partial x^{l}}-\frac{\partial \Gamma_{i l}^{l}}{\partial x^{k}}\right]\right\} .
$$

In Galilean coordinates (where the coefficients $(\Gamma)$ are zero) one finds

$$
\left\{\begin{array}{c}
g^{i k} \delta R_{i k}=g^{i k}\left(\frac{\partial}{\partial x^{l}} \delta \Gamma_{i k}^{l}-\frac{\partial}{\partial x^{j}} \delta \Gamma_{i k}^{j}\right)=g^{i k} \frac{\partial}{\partial x^{l}} \delta \Gamma_{i k}^{l}-g^{i l} \frac{\partial}{\partial x^{l}} \delta \Gamma_{i k}^{k}=\left(\frac{\partial w^{l}}{\partial x^{l}}\right)=\operatorname{div}(\vec{w}) \\
w^{l}=g^{i k} \delta \Gamma_{i k}^{l}-g^{i l} \delta \Gamma_{i k}^{k}
\end{array}\right\} .
$$

Into arbitrary coordinates [10] one finds:

$$
g^{i k} \delta R_{i k}=\left(\frac{1}{\sqrt{-g}}\right)\left[\frac{\partial\left(w^{l} \sqrt{-g}\right)}{\partial x^{l}}\right]
$$


Here the second term is the covariant derivative of vector $(w)$ or divergence of $w$.

We note that

$$
\left[\int\left(g^{i k} \delta R_{i k}\right)(\sqrt{-g}) \mathrm{d} \Omega\right]=\int\left(\frac{\partial\left(w^{l} \sqrt{-g}\right)}{\partial x^{l}}\right) \mathrm{d} \Omega=\int_{\partial \Omega} \underline{\vec{w}} \cdot \mathrm{d} \vec{S}
$$

where we has used the Stokes's theorem to border surface of volume $\Omega$, with $S=$ $\partial \Omega$.

If $S$ is the border surface wrapping the varied curves, for Gauss theorem, the integral would be zero or the vector flow $(w)$ is zero

$$
\Phi(\vec{w})_{S^{\circ}}=\int_{S^{\circ}} \underline{\vec{w}} \cdot \mathrm{d} \vec{S}=0 .
$$

If happen in this way then we obtain (varying the action $S$ ) the equation of standard cosmology

$$
\delta S_{g}=\left[\int\left(R_{i k}-\frac{1}{2} g^{i k} R\right) \delta g^{i k}(\sqrt{-g}) \mathrm{d} \Omega\right] .
$$

So seems that to investigate about physical mean of the (div $w$ ) is not important because this vanishes in $\delta S_{g}$. Therefore if we prove that into expanding space the flow of $(w)$ is not more zero be when it is going through the border surface $(S)$, then it is essential find the physical mean of the flow of $(w)$. Besides being the (div $w$ ) not zero would add another term in the cosmologic equation without $(\Lambda)$. Therefore we ask us what happens into action $S_{U}$ if we admit the mass-space increasing in the Universe with expansion ( $\underline{U}$ ). At cause of the expansion, in this $(\underline{U})$ the superior extreme could be variable at any instant of cosmic time, with the consequence of having the $\left(\delta g_{i k}\right)$ not more zero. In the cosmology with mass-space increasing this is equivalent to admit that the flow of mass-space would make not zero the $(\operatorname{div} w)$. For incorporate the expansion into variational principle we put the superior extreme of generalized trajectory not more in $\underline{t}_{2}$ but in $\left(\underline{t}_{2}+\mathrm{d} \underline{t}\right)$, so making we put inside the increased space during the integration in range $\left[\underline{t}_{1}\right.$, $\left.\underline{t}_{2}\right]$. So we will have a space-flow not zero through the border surface $S_{2}\left(\underline{t}_{2}\right)$ but zero through the extreme border surface $S_{2}^{\prime}\left(\underline{t}_{2}+\mathrm{d} t\right)$ which coincides with the surface $S_{2}\left(\underline{t}_{2}\right)$ but in $\underline{U}$ with expansion. In this way during the "evolution" of $\underline{U}$ from $\underline{t}_{1}$ to $\left(\underline{t}_{2}+\mathrm{d} \underline{t}\right)$ we can put in $S_{U}$ action the space expansion. All this is feasible if in the variations of generalized coordinates $\left(\delta q_{j} \equiv \delta g_{i k}\right)$ that build the varied curves of the "motion" of physical system we admit the coordinate variations for expansion $\left(\delta g_{i k}\right)$. We will have a linear combination of variations: $\left(\delta g_{i k}\right)=\left(\delta g_{i k}\right)$ $+\left(\underline{\delta g_{i k}}\right)$.

In this way we can fix the superior extremis of range of integration and to see the trajectory variations as linear combination of two possible variations.

We will have the following Figure 12.

The superior extreme of the action integral is not more in $\underline{t}_{2}$,because there are not only virtual variations $\left(\delta g_{i k}\right)_{\text {var }}$, but also the $\left(\delta g_{i k}\right)_{\exp }$ variations caused by 


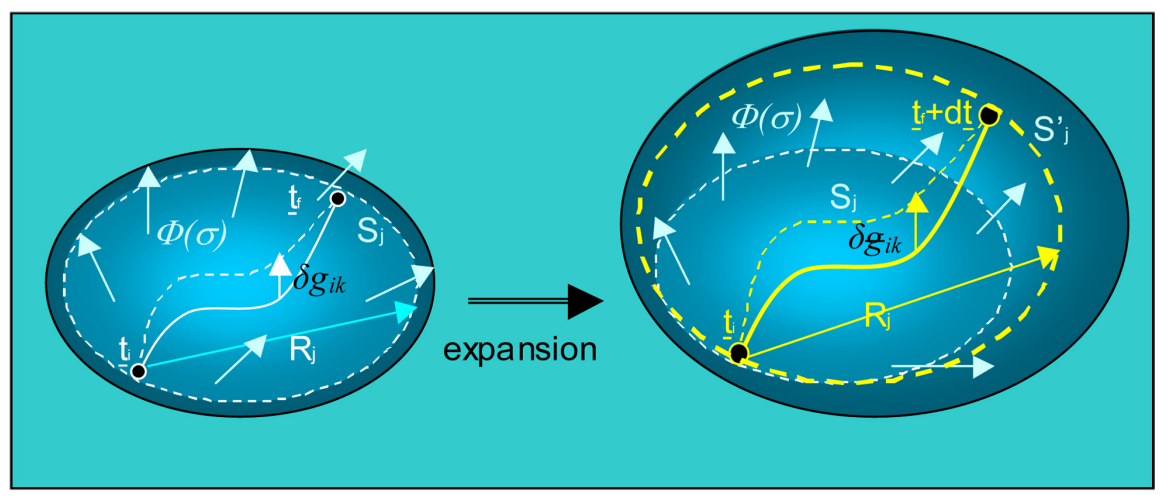

Figure 12. Metric variation for expansion.

expansion; the new superior extreme will be $\left(\underline{t}_{2}+\mathrm{d} t\right)$ where the global $\left(\delta g_{i k}\right)$ are zero.

Note that the $\left(\delta g_{i k}\right)$ can be equivalent to $\left(\delta g_{i k}\right)_{\mathrm{var}}$ if the $x_{i}$-coordinates of any reference frame be expressed by scale factor.

$$
\delta g_{i k}=\left(\delta g_{i k}\right)_{\mathrm{var}}+\left(\delta g_{i k}\right)_{\mathrm{exp}} \Rightarrow \delta g_{i k} \equiv\left\{\begin{array}{c}
g_{i k} \\
x \rightarrow a(t) x
\end{array}\right\} .
$$

Then we can take in consideration that the integral with $(\delta R)$, you see the Equation (5.10), is not more zero inside time-range $\left[\underline{t}_{1}, \underline{t}_{2}\right]$. Instead into range $\left[\underline{t}_{1}, \underline{t}_{2}+\right.$ $\mathrm{d} t]$ it is:

$$
\begin{aligned}
\delta S_{g} & =\left[\int\left(R_{i k}-\frac{1}{2} g_{i k} R\right) \delta g^{i k}(\sqrt{-g}) \mathrm{d} \Omega\right]+\left[\int\left(g^{i k} \delta R_{i k}\right)(\sqrt{-g}) \mathrm{d} \Omega\right] \\
& =\left[\int\left(R_{i k}-\frac{1}{2} g_{i k} R\right) \delta g^{i k}(\sqrt{-g}) \mathrm{d} \Omega\right]+\int\left(\frac{\partial\left(w^{l} \sqrt{-g}\right)}{\partial x^{l}}\right) \mathrm{d} \Omega .
\end{aligned}
$$

Last term can be:

$$
\left(\frac{\partial\left(w^{l} \sqrt{-g}\right)}{\partial x^{l}}\right)=\left[w^{l} \frac{\partial(\sqrt{-g})}{\partial x^{l}}+(\sqrt{-g}) \frac{\partial\left(w^{l}\right)}{\partial x^{l}}\right]
$$

then last integral is

$$
\int\left(\frac{\partial\left(w^{l} \sqrt{-g}\right)}{\partial x^{l}}\right) \mathrm{d} \Omega=\int\left[w^{l} \frac{\partial(\sqrt{-g})}{\partial x^{l}}\right] \mathrm{d} \Omega+\int\left[(\sqrt{-g}) \frac{\partial w^{l}}{\partial x^{l}}\right] \mathrm{d} \Omega .
$$

The variation of action into time-range $\left[\underline{t}_{1}, \underline{t}_{2}+\mathrm{d} t\right]$ is

$$
\begin{aligned}
\delta S_{g}= & {\left[\int\left(R_{i k}-\frac{1}{2} g_{i k} R\right) \delta g^{i k}(\sqrt{-g}) \mathrm{d} \Omega\right] } \\
& +\int\left[w^{l} \frac{\partial(\sqrt{-g})}{\partial x^{l}}\right] \mathrm{d} \Omega+\int\left[(\sqrt{-g}) \frac{\partial w^{l}}{\partial x^{l}}\right] \mathrm{d} \Omega .
\end{aligned}
$$

Carrying the superior extreme in $\left[\underline{t}_{2}+\mathrm{d} t\right]$ the third term is zero because is a integral of a divergence term of which the flow is zero through extreme surface 
$S_{2}^{\prime}$. Then to end write

$$
\delta S_{g}=\left[\int\left(R_{i k}-\frac{1}{2} g_{i k} R\right) \delta g^{i k}(\sqrt{-g}) \mathrm{d} \Omega\right]+\int w^{l} \frac{\partial(\sqrt{-g})}{\partial x^{l}} \mathrm{~d} \Omega .
$$

Having that $[10]$

$$
\frac{\delta(\sqrt{-g})}{(\sqrt{-g})}=-\frac{1}{2} g_{i k} \delta g^{i k}
$$

it follows that

$$
\begin{aligned}
\int\left[w^{l} \frac{\partial(\sqrt{-g})}{\partial x^{l}}\right] \mathrm{d} \Omega & =\int w^{l}\left[(\sqrt{-g})\left(\frac{1}{\delta x_{l}}\right)(\delta(\sqrt{-g}))\right] \mathrm{d} \Omega \\
& =\int\left[\frac{w^{l}}{\Delta x^{l}}\right]\left(\delta g_{i k}\right)(\sqrt{-g}) \mathrm{d} \Omega \\
& =\int\left[\frac{w^{l}}{\Delta x^{l}}\right]\left[\frac{-1}{2} g^{i k}\right]\left(\delta g_{i k}\right)(\sqrt{-g}) \mathrm{d} \Omega \\
& =\int\left[\frac{-w^{l}}{2 \Delta x^{l}} g^{i k}\right]\left(\delta g_{i k}\right)(\sqrt{-g}) \mathrm{d} \Omega
\end{aligned}
$$

or with whole details

$$
\begin{aligned}
\int\left[w^{l} \frac{\partial(\sqrt{-g})}{\partial x^{l}}\right] \mathrm{d} \Omega & =\int w^{l}\left[\frac{-1}{2 \sqrt{-g}}\left(\frac{\partial g}{\partial x^{l}}\right)\right] \mathrm{d} \Omega=\int w^{l}\left[\left(\frac{-1}{2 \sqrt{-g}}\right)\left(2 g \Gamma_{l i}^{i}\right)\right] \mathrm{d} \Omega \\
& =\int-w^{l}\left[\left(\Gamma_{l i}^{i}\right)\right](\sqrt{-g}) \mathrm{d} \Omega=\int-w^{l}\left[\frac{1}{2} g^{i k}\left(\frac{\partial g_{i k}}{\partial x^{l}}\right)\right](\sqrt{-g}) \mathrm{d} \Omega \\
& =\int w^{l}\left[\frac{-1}{2} g^{i k}\left(\frac{\partial g_{i k}}{\partial x^{l}}\right)\right]\left(\frac{\Delta x^{l}}{\Delta x^{l}}\right)(\sqrt{-g}) \mathrm{d} \Omega \\
& =\int w^{l}\left[\frac{-1}{2} g^{i k}\right]\left(\delta g_{i k} \frac{1}{\Delta x^{l}}\right)(\sqrt{-g}) \mathrm{d} \Omega \\
& =\int\left[\frac{-w^{l}}{2 \Delta x^{l}} g^{i k}\right]\left(\delta g_{i k}\right)(\sqrt{-g}) \mathrm{d} \Omega .
\end{aligned}
$$

Replacing in the action variation we obtain

$$
\begin{aligned}
\delta S_{g} & =\left[\int\left(R_{i k}-\frac{1}{2} g_{i k} R\right) \delta g^{i k}(\sqrt{-g}) \mathrm{d} \Omega\right]+\int\left[\frac{-w^{l}}{2 \mathrm{~d} x^{l}} g^{i k}\right]\left(\delta g_{i k}\right)(\sqrt{-g}) \mathrm{d} \Omega \\
& =\int\left[\left(R_{i k}-\frac{1}{2} g_{i k} R\right)+\left(\frac{-w^{l}}{2 \Delta x^{l}} g^{i k}\right)\right]\left(\delta g_{i k}\right)(\sqrt{-g}) \mathrm{d} \Omega .
\end{aligned}
$$

If we put

$$
W=\left(\frac{1}{2}\right)\left(\frac{w^{l}}{\Delta x^{l}}\right)
$$

Will be 


$$
\begin{aligned}
\delta S_{g} & =\left[\int\left(R_{i k}-\frac{1}{2} g_{i k} R\right) \delta g^{i k}(\sqrt{-g}) \mathrm{d} \Omega\right]-\int W g_{i k} \delta g^{i k}(\sqrt{-g}) \mathrm{d} \Omega \\
& =\int\left\{\left[\left(R_{i k}-\frac{1}{2} g_{i k} R\right)-W g_{i k}\right] \delta g^{i k}(\sqrt{-g})\right\} \mathrm{d} \Omega .
\end{aligned}
$$

Change $(W)$ scalar in $(\Lambda)$,. We will have that $[\operatorname{div} w]=\Lambda g_{i k}$

$$
\begin{aligned}
\delta S_{g} & =\left[\int\left(R_{i k}-\frac{1}{2} g_{i k} R\right) \delta g^{i k}(\sqrt{-g}) \mathrm{d} \Omega\right]+\int W g_{i k} \delta g^{i k}(\sqrt{-g}) \mathrm{d} \Omega \\
& =\int\left\{\left[\left(R_{i k}-\frac{1}{2} g_{i k} R\right)-\Lambda g_{i k}\right] \delta g^{i k}(\sqrt{-g})\right\} \mathrm{d} \Omega .
\end{aligned}
$$

This equation is coincident with Einstein cosmologic equation, where $\Lambda g_{i k}$ had been introduced for make universe in way stationary. In this cosmologic model $(\Lambda)$ is not obtained "ad hoc" as in the Einstein model; in fact here we have:

$$
\Lambda=\left(\frac{1}{2}\right)\left(\frac{w^{l}}{\Delta x^{l}}\right) .
$$

To compare with $((4.21)$ or $(4.23))$ where it is had: $\left[\Lambda=2 / \lambda^{2}\right]$. Comparing with the Equation (5.30):

$$
\left\{\left(\frac{1}{2}\right)\left(\frac{w^{l}}{\Delta x^{l}}\right)=\left(\frac{2}{\hbar^{2}}\right)\right\} \Rightarrow\left\{w^{l}=4 \frac{\Delta x^{l}}{\hbar^{2}}\right\} .
$$

If the $\mathrm{d} x$ is the increase of space along the IQuO-chain then it is:

$$
\left\{\vec{w} \cdot \mathrm{d} \vec{x}=w^{l} \mathrm{~d} x_{l}=4 \frac{\mathrm{d} x^{l} \mathrm{~d} x_{l}}{\hbar^{2}}=4 \frac{g^{l k} \mathrm{~d} x_{k} \mathrm{~d} x_{l}}{\hbar^{2}}=\frac{16 \pi g^{l k} \mathrm{~d} S_{k l}}{f_{c}}=(16 \pi) \frac{\mathrm{d} S}{f_{c}}\right\}
$$

where $\mathrm{d} S$ represents the increase of space ( or surface increase); if $\left(\lambda \equiv \lambda_{c}\right)$ then $S_{c}$ will come called "Compton's Surface". The physical meaning of the vector $(w)$ it is so connected to the space flow $\Phi(\underline{w})$ (you see the Equation (5.14)) which "builds space" in the lattice universe $U_{\dot{r}}$. We will call the $\Phi(\underline{w})$ space flow or incremental ratio of space. Recall the vector form from Equation (5.12):

$$
w^{l}=g^{i k} \delta \Gamma_{i k}^{l}-g^{i l} \delta \Gamma_{i k}^{k} .
$$

What is the term $\left(g^{i k} \Gamma_{i k}^{l}\right)$ ? If $\left(\Gamma_{i k}^{l} A_{k} \mathrm{~d} x^{l}\right)$ is variation $\left[\Delta_{T} A\right]$ which the vector A has in following parallel transport along a curve $(\gamma)$, the term $\left(\delta \Gamma_{i k}^{l} A_{k} \mathrm{~d} x^{l}\right)$ instead identifies the difference between the two vectors obtained by the same carrier $\boldsymbol{A}$ for parallel transport along the curves $(\gamma)$ and $(\gamma)$, seed as variation of $(\gamma)$ or $\left[\delta_{T\left(\gamma, \gamma^{\prime}\right)} \boldsymbol{A}=\left(\Delta_{T} \boldsymbol{A}\right)_{\gamma^{\prime}}-\left(\Delta_{T} \boldsymbol{A}\right)_{\gamma}\right]$; with: $\left[\delta_{T\left(\gamma, \gamma^{\prime}\right)} \boldsymbol{A}=\delta \Gamma_{i k}^{l} A_{k} \mathrm{~d} x^{l}\right]$.

Note [4] that in increasing space, during parallel transport of vector A along the $(\gamma)$ curve or $\left(A \rightarrow A_{T}\right)$ we should add an ulterior variation for expansion $\left(A \rightarrow A_{T}^{\prime}\right)$ in Figure 13.

Observing the $(w)$ form in the (5.13) we could realize that the physical meaning of the vector $\mathrm{w}$ can be to describe the variation of the metric due to variation of any vector A for effect of the expansion.

This variation can be achieved by carrying out the scalar product of the vector 


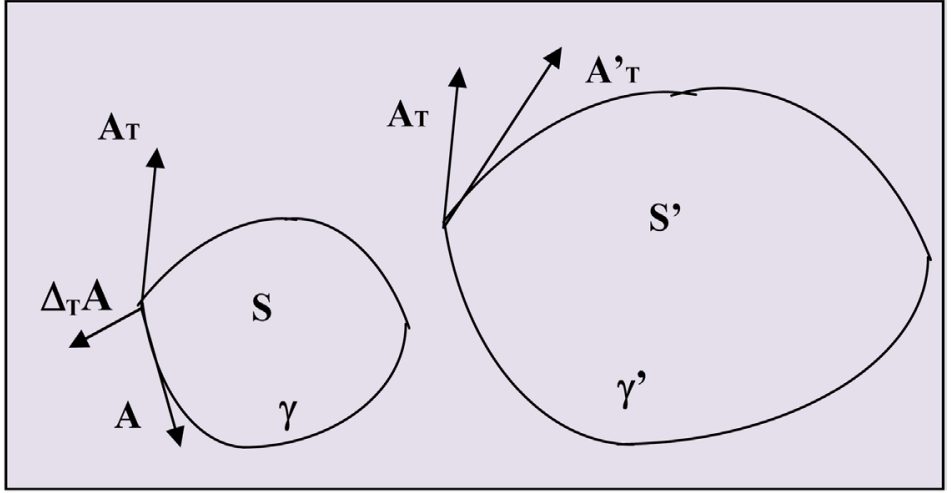

Figure 13. Ulterior variation of vector $A$ for expansion.

$\boldsymbol{A}$ with the ( $w$ ) vector. Looking better the (5.14) and the physical dimension of $w$ $\left([w]=L^{-1}\right)$ we guess that the vector $\mathrm{w}$ is a generator of the amount of "line" along a "chain" IQuO coupled which induces ulterior variations of the metric, expressed by the element $\left(\delta \Gamma_{i k}^{l}\right)$. Note that along a chain IQuO where is going a photon the arising of a space cell involves, for adapting to the new length of $\mathrm{IQuO}$, the increasing of the period of oscillation associated with it (delay). Well this "delay" implies a metric variation (variation of the time component of the 4 -vector associated with the photon).

Calculating the infinitesimal $(w)$-flow through the surface $S$ with boundary $(\gamma)$ :

$$
\begin{aligned}
w^{l} \mathrm{~d} S_{l} & =g^{i k} \delta \Gamma_{i k}^{l} \mathrm{~d} S_{l}-g^{i l} \delta \Gamma_{i k}^{k} \mathrm{~d} S_{l}=\delta \Gamma_{i k}^{l} g^{i k} \mathrm{~d} S_{l}-\delta \Gamma_{i k}^{k} g^{i l} \mathrm{~d} S_{l} \\
& =\delta \Gamma_{i k}^{l} g^{i k}(\mathrm{~d} x)_{l}^{2}-\delta \Gamma_{i k}^{k} g^{i l}(\mathrm{~d} x)_{l}^{2} \\
& =\delta \Gamma_{i k}^{l}\left(g^{i k} \mathrm{~d} x_{l} \mathrm{~d} x_{l}\right)-\delta \Gamma_{i k}^{k}\left(g^{i l} \mathrm{~d} x_{l} \mathrm{~d} x_{l}\right) \\
& =\delta \Gamma_{i k}^{l}\left(g^{i k} g_{l k} \mathrm{~d} x^{k} \mathrm{~d} x_{l}\right)-\delta \Gamma_{i k}^{k}\left(g^{i l} g_{l k} \mathrm{~d} x^{k} \mathrm{~d} x_{l}\right) \\
& =\delta \Gamma_{i k}^{l}\left(\delta_{l}^{i} \mathrm{~d} x^{k} \mathrm{~d} x_{l}\right)-\delta \Gamma_{i k}^{k}\left(\delta_{k}^{i} \mathrm{~d} x^{k} \mathrm{~d} x_{l}\right) \\
& =\delta \Gamma_{i k}^{l}\left(\delta_{l}^{i} \mathrm{~d} x^{k}\right)\left(\mathrm{d} x_{l}\right)-\left(\delta \Gamma_{i k}^{k} \delta_{k}^{i}\right)\left(\mathrm{d} x_{l}\right) \\
& =\delta \Gamma_{i k}^{l}\left(\delta_{l}^{i} \mathrm{~d} x^{k} \mathrm{~d} x_{l}\right)-0 \\
& =\delta \Gamma_{i k}^{i}\left(\delta_{l}^{i} \mathrm{~d} x^{k}\right)\left(\mathrm{d} x_{l}\right)=\delta \Gamma_{i k}^{i}\left(\delta x^{k}\right)\left(\mathrm{d} x_{i}\right) .
\end{aligned}
$$

Then (you see the Equation (5.13)) we obtain that the $(w)$ flow is generating of increasing space $(\delta x)$ with metric variation $\delta \Gamma$. We have unveiled the physical meaning of $w$, as well as that $\Lambda$ related to it.

\subsection{Tensor $T_{i k}$ with Density Variable}

Let us pass to consider, now, the mass flow which associated with space flow. The Action of mass-energy, in curvilinear coordinates will be

$$
\delta S_{m}=\delta \int £_{m}\left(g_{i k}, \partial g_{i k}\right)(\sqrt{-g}) \mathrm{d} \Omega .
$$

The variation of $S$ action will be 


$$
\delta S_{m}=\delta \int £_{m}(\sqrt{-g}) \mathrm{d} \Omega=0 .
$$

Varying the $S_{m}$, one gets [10]

$$
\delta S_{m}=\frac{1}{c} \int_{S}\left[\frac{\partial E(\sqrt{-g})}{\partial g^{i k}}-\frac{\partial}{\partial x^{i}} \frac{\partial E(\sqrt{-g})}{\partial\left(\frac{\partial g^{i k}}{\partial x^{l}}\right)}\right] \delta g^{i k} \mathrm{~d} \Omega .
$$

Setting

$$
\frac{1}{2}(\sqrt{-g}) T_{i k}=\left[\frac{\partial £(\sqrt{-g})}{\partial g^{i k}}-\frac{\partial}{\partial x^{i}} \frac{\partial £(\sqrt{-g})}{\partial\left(\frac{\partial g^{i k}}{\partial x^{l}}\right)}\right] .
$$

It follows

$$
\delta S_{m}=\delta \int £_{m}(\sqrt{-g}) \mathrm{d} \Omega=\frac{1}{2 c} \int_{S} T_{i k} \delta g^{i k}(\sqrt{-g}) \mathrm{d} \Omega .
$$

Adding this term into variation of the "spatial" action $S_{g}$, one obtains the Einstein cosmologic equation

$$
\begin{aligned}
\delta S_{g}+\delta S_{m}= & \left(-\frac{c^{3}}{16 \pi G}\right) \int\left(R_{i k}-\frac{1}{2} g_{i k} R\right) \delta g^{i k}(\sqrt{-g}) \mathrm{d} \Omega \\
& +\left(\frac{1}{2 c}\right) \int T_{i k} \delta g^{i k}(\sqrt{-g}) \mathrm{d} \Omega=0 .
\end{aligned}
$$

Nevertheless it is necessary making symmetrical $T_{i k}$; we can follow two physical equivalent way.

The first way implies that $T_{i k}$ is transformed as

$$
T^{i k} \Rightarrow T^{i k}+\left(\frac{\partial \psi^{i k l}}{\partial x^{l}}\right)=T^{i k}+\operatorname{div} \psi^{i k l}
$$

Now the tensor $T_{i k}$ is symmetrical but not univocal (the energy conservation law is not valid because this pass from energy $(T)$ to $T^{\prime}=T+\Delta T$ with $(\Delta T \Leftrightarrow$ $\operatorname{div} \psi$ ). Though [10] the 4-impulse (p) can be instead univocal if we set that:

$$
\int_{S}\left(\frac{\partial \psi^{i k l}}{\partial x^{l}}\right) \mathrm{d} S_{k}=\int_{S} \psi^{i k l} \mathrm{~d} S=0 .
$$

The $\psi$ flow is zero if $S$ is the border surface wrapping the universe: so that the energy-impulse conservation is reestablished. The div $\psi$ is very important not only because $T_{i k}$ becomes a symmetrical tensor but also because it could justify the existence of a mass-energy flow which crosses the $S(t)$ surface. All this be if (div $\psi \neq 0$ ) on the $S(t)$ surface. However, as it has already been made for space flow, we consider (you see the Figure 12) the surface $S$ as border surface in $t \rightarrow S(t)$, while $S^{\prime}$ is a border surface in $(t+d t) \rightarrow S^{\prime}(t+d t)$.

Then the $\psi$ function can describe a mass-flow crossing the $S(t)$ surface but not $S(t+d t)$. 
All this is admissible if exist an additional physical system $(\Theta)$ (with "action" function $S_{\Theta}$ ) which is source of mass-flow crossing $S^{\prime}(t)$.

This admission cannot be "ad hoc", since the $(\Theta)$ is the source of the mass-space flow which causes the expansion and its acceleration, completing so the EF cosmologic theory (you see Einstein [4]).

Instead of considering an action $S_{U}$ on the system Universe $U$ with variable extremes due to expansion, we can fix the extremes of the generalized trajectory not in $(t)$ but in $(t+d t)$; in such way we can affirm that there is a "mass flow" $[\Phi(\mu)]$ which crosses the border surface $S(t)$ but not the surface $S^{\prime}(t+d t)$. In this way during the motion of $S_{U}$ system from $(t)$ to $(t+d t)$ along generalized trajectory we can add another $S_{\Theta}$ action in the $S_{U}$ action which is associated to mass-flow in any $\underline{t}_{i} \in\left[\underline{t}_{0},(\underline{t}+d \underline{t})\right]$. So we will have the transition:

$\left[\left(S_{U}=S_{g}+S_{m}\right) \Leftrightarrow \partial S_{U}=0\right] \rightarrow\left[\left(S_{U^{*}}=S_{g}+S_{m}+S_{\Theta}\right) \Leftrightarrow \partial S_{U^{*}}=0\right]$.

The second way for making $(T)$ in form symmetrical it starts by transformation of coordinates $\left(x^{i}\right)$ involving the metric $\left(g_{i k}\right)$ or

$$
\left\{\begin{array}{c}
x^{i} \Rightarrow x^{\prime i}=x^{i}+\xi^{i} \\
g^{i k}\left(x^{l}\right) \Rightarrow g^{i k}\left(x^{\prime \prime}\right) \approx g^{i k}\left(x^{l}\right)+\delta g^{i k} \\
\delta g^{i k}=g^{i m}\left(\frac{\partial \xi^{k}}{\partial x^{m}}\right)+g^{i k}\left(\frac{\partial \xi^{i}}{\partial x^{l}}\right)
\end{array}\right\} .
$$

A novelty could be that to include the transformations describing "the expansion of the space", through the presence of a scale factor $a(t)$ :

$$
x^{i} \Rightarrow x^{i}=x^{i}+a(\underline{t}) \xi^{i}=x^{i}+\xi^{i}(\underline{t}) .
$$

As we have already stated the expansion determine a metric variation $\rightarrow\left(\delta g_{i k}\right)$. Having the equivalence mass-flow $\Leftrightarrow$ space-flow we can affirm that the variation $\left(\delta g_{\mathrm{k}}\right)$ implies mass variation.

Therefore, for the energy conservation law, the mass flow has a source (the $\Theta$-underground) which is expressed as $S_{\Theta}$. Now we will have the Universe system $\left[U^{*}=U+\Theta\right]$. We consider for simplicity that the system of galaxies of the universe $\mathrm{U}$ is system mass-energy analogous to a system of particles (gas).

The [4] density of 4-impulse $\left(u^{i}\right)$ will be: $\left(\rho u^{i}\right) \equiv\left(\rho u^{0}, \rho u\right)$. We will have:

$$
T^{i k}=\rho c u^{i} u^{k}\left(\frac{\mathrm{d} s}{\mathrm{~d} t}\right)
$$

calculating the derivative $(\partial T)$, will be

$$
\frac{\partial T_{i}^{k}}{\partial x^{k}}=\frac{\partial}{\partial x^{k}}\left(\rho c u^{i} \frac{\mathrm{d} x^{k}}{\mathrm{~d} t}\right)=c u^{i} \frac{\partial}{\partial x^{k}}\left(\rho \frac{\mathrm{d} x^{k}}{\mathrm{~d} t}\right)+\rho c\left(\frac{\mathrm{d} x^{k}}{\mathrm{~d} t} \frac{\partial u_{i}}{\partial x^{k}}\right) .
$$

In such a case the $\rho$ density of the galaxies gas could be no constant and then would have that

$$
\frac{\partial T_{i}^{k}}{\partial x^{k}}=c u^{i} \frac{\partial}{\partial x^{k}}\left(\rho \frac{\mathrm{d} x^{k}}{\mathrm{~d} t}\right)+\rho c\left(\frac{\mathrm{d} u_{i}}{\mathrm{~d} t}\right)=c u^{i}\left[\frac{\mathrm{d} \rho}{\mathrm{d} t} \frac{\mathrm{d} x^{k}}{\mathrm{~d} t}+\rho \frac{\partial}{\partial x^{k}} \frac{\mathrm{d} x^{k}}{\mathrm{~d} t}\right]+\rho c\left(\frac{\mathrm{d} u_{i}}{\mathrm{~d} t}\right) .
$$


Or

$$
\frac{\partial T_{i}^{k}}{\partial x^{k}}=c u^{i} \frac{\partial}{\partial x^{k}}\left(\rho \frac{\mathrm{d} x^{k}}{\mathrm{~d} t}\right)+\rho c\left(\frac{\mathrm{d} u_{i}}{\mathrm{~d} t}\right)=c u^{i}\left[\operatorname{div}_{i}\right]+\rho c\left(\frac{\mathrm{d} u_{i}}{\mathrm{~d} t}\right) .
$$

Will be

$$
\frac{\partial T_{i}^{(p) k}}{\partial x^{k}}=c u^{i}[\operatorname{div} J]^{(p)}+\rho c\left(\frac{\mathrm{d} u_{i}}{\mathrm{~d} t}\right)^{(p)}=-\frac{\partial T_{i}^{(\Theta) k}}{\partial x^{k}} .
$$

Here $T_{i k}^{(\Theta)}$ is energy density tensor of $(\Theta)$.

However the $(\Theta)$ cannot influence the 4-velocity $\left(u^{i}\right)$ because it is an "inert" physical object or a not-field (you see the par. 4.3); therefore it is $\left[\mathrm{d} u^{i} / \mathrm{d} t=0\right]$ and thus it happens:

$$
\frac{\partial T_{i}^{(p) k}}{\partial x^{k}}=c u^{(p) i}\left[\operatorname{div}^{(p)} J\right]=-\frac{\partial T_{i}^{(\Theta) k}}{\partial x^{k}} .
$$

You should seek the form of $T_{i k}^{(\Theta)}$. The $(\Theta)$ is the not-field and therefore this is made up of a set of uncoupled oscillators having quanta; this system could be analogous to system of particles like a "gas". We can set $T^{(\Theta)} \equiv T^{\text {(gas) }} \equiv T$; the tensor $T$ would be similar to $T$ tensor of the galactic gas in GR (you see [4]), so we have

$$
(T)_{\Theta}=T^{i k}=\left(P_{\Theta}+\varepsilon_{\Theta}\right) u^{i} u^{k}-P_{\Theta} g^{i k} .
$$

However $(\Theta)$ is an inactive physical object and then this cannot influence on the 4-impulse $\left(p^{t}\right)$ : so we have that $\left(P_{\Theta}=0\right)$. Then we could think that $(\Theta)$ is just a "liquid" and no gas, having so that:

$$
\mp^{i k}=\left(\varepsilon_{\Theta}\right) u^{i} u^{k} .
$$

Of which the derivative is:

$$
\begin{aligned}
\frac{\partial T_{i}^{k}}{\partial x^{k}} & =\frac{\partial}{\partial x^{k}}\left(\rho_{\Theta} c^{2} u^{i} \frac{\mathrm{d} x^{k}}{\mathrm{~d} t}\right)=c^{2} u^{i} \frac{\partial}{\partial x^{k}}\left(\rho \frac{\mathrm{d} x^{k}}{\mathrm{~d} t}\right)+\rho c^{2}\left(\frac{\mathrm{d} x^{k}}{\mathrm{~d} t} \frac{\partial u_{i}}{\partial x^{k}}\right) \\
& =c^{2} u^{i} \frac{\partial}{\partial x^{k}}\left(\rho_{\Theta} \frac{\mathrm{d} x^{k}}{\mathrm{~d} t}\right)=c u^{i}\left(\operatorname{div} J_{\Theta}\right)
\end{aligned}
$$

equating the derivative of two tensor $T^{(p)}, T^{(\Theta)}$; it will be

$$
\left[\operatorname{div} J_{i}^{m}\right]=-\left[\operatorname{div} J_{i}^{(\Theta)}\right] \text {. }
$$

It follows

$$
\frac{\partial}{\partial x^{k}}\left(\rho_{m} \frac{\mathrm{d} x^{k}}{\mathrm{~d} t}\right)_{U}=-\frac{\partial}{\partial x^{k}}\left(\rho_{\Theta} \frac{\mathrm{d} x^{k}}{\mathrm{~d} t}\right)_{\Theta}
$$

and also

$$
\begin{aligned}
& \left(\frac{\rho_{m^{\prime}} u^{k}-\rho_{m} u^{k}}{\left(x^{\prime}-x\right)}\right)=-\left(\frac{\rho_{\Theta} u^{k}-\rho_{\Theta} u^{k}}{\left(x^{\prime}-x\right)}\right) \\
& \Rightarrow\left(\rho_{m^{\prime}} u_{\left(m^{\prime}\right)}^{k}-\rho_{m} u_{\left(m^{\prime}\right)}^{k}\right)=-\left(\rho_{\Theta} u_{(\Theta)}^{\prime k}-\rho_{\Theta} u_{(\Theta)}^{\prime k}\right) .
\end{aligned}
$$


Because the incoming mass into $U$ coincides with the exiting mass from $\Theta$, it happens that the velocity $\left(u_{\Theta}\right)$ of the exiting mass from $\Theta$ coincides with the velocity of the incoming mass flow in the universe or $\left(u_{m}\right) \rightarrow\left(u_{\Theta} \equiv u_{m}\right)$; it follows then:

$$
\left(\rho_{m^{\prime}}-\rho_{m}\right)_{\Delta t}=-\left(\rho_{\Theta^{\prime}}-\rho_{\Theta}\right)_{\Delta t} .
$$

In such way the mass density of Universe is variable to pass of cosmic time or $\rho_{m}=f(\underline{t})$.

As it will see in future, the form of the $\rho_{m}$ function is determined by conditions which derives from process of transfer of quanta from $\Theta$ to $U$.

Therefore we can introduce in $T^{(p)}$ a variable mass density in time. In this way the action $S_{\Theta}$ is expressed by an energy-mass tensor $(T)$ with variable mass density.

We can have $\left[S_{U}=S_{g}+\left(S_{m}+S_{\Theta}\right) \equiv S_{g}^{\prime}+S_{m}^{\prime}\right]$.

Here $S_{m}^{\prime}$ is the action of mass-energy to variable mass density and $S_{g}^{\prime}$ is the action of S-T field with expansion. Therefore is evident that the Universe $U$ has a variable metric because there is a mass-space flow due to the presence of the not-field $\Theta$. The variation of global action is

$$
\begin{aligned}
\delta U=\delta S_{g}+\delta S_{m}= & \int\left\{\left[\left(R_{i k}-\frac{1}{2} g_{i k} R\right)-\Lambda g_{i k}\right] \delta g^{i k}(\sqrt{-g})\right\} \mathrm{d} \Omega \\
& -\int \chi \underline{T}_{i k} \delta g^{i k}(\sqrt{-g}) \mathrm{d} \Omega=0 .
\end{aligned}
$$

Here $\left(\underline{T}_{i k}\right)$ is the tensor with variable mass density having the same form of tensor $\left(\underline{T}_{i k}\right)$ into cosmologic equation. So we obtain a new equation in which are present both the cosmologic parameter $(\Lambda)$ and both the mass-energy tensor $\left(\underline{T}_{i k}\right)$ with variable mass density, or due

$$
\left[\left(R_{i k}-\frac{1}{2} g_{i k} R\right)-\Lambda g_{i k}\right]=\chi \underline{T}_{i k} .
$$

For the equivalence mass-space and for the pressure of mass-space flow we can move $(\Lambda)$ to second member of the equation associating it at mass-energy tensor; then we will have that

$$
\left\{\left(R_{i k}-\frac{1}{2} g_{i k} R\right)=\chi \underline{T}_{i k}+\Lambda g_{i k}\right\}_{i k}
$$

if it is $T_{i k}^{*}=T_{i k}+\Lambda_{i k} ;$ it follows

$$
\left\{\left(R_{i k}-\frac{1}{2} g_{i k} R\right)=\underline{T}_{i k}^{*}\right\}_{i k} .
$$

This is called: cosmologic equation with variable metric.

\subsection{The Solutions of the Cosmological Equation}

In a universe where the mass implies space-time and vice versa we cannot think of putting at "zero" all the sources of the "universe" field or put $\left[T_{i k}=0\right]$. On the 
contrary in literature it is put $\left[T_{i k}=0\right]$ for thinking of getting the vacuum energy of Universe field; you see the relation:

$$
\left\{\left(R_{i k}-\frac{1}{2} g_{i k} R\right)=\Lambda g_{i k}\right\}_{i k}
$$

here $\Lambda_{i k}$ would be the vacuum energy tensor. Instead in our cosmological model must be always $\underline{T}_{i k} \neq 0$. Yet also here we have associated a pressure $\left(P_{\exp }\right)$ to the flow of space-mass existing in the universe field $\Xi_{U}$. Therefore, we will associate with $\left(P_{\exp }\right)$ an energy $\left(\varepsilon_{\exp }\right)$. We will have that:

$$
\varepsilon_{\exp }=3 P_{\exp }=\left(\frac{3 \Lambda c^{4}}{8 \pi G}\right)=\left(\frac{3 \Lambda}{\chi}\right)
$$

we will write

$$
\left\{\left(R_{i k}-\frac{1}{2} g_{i k} R\right)=\chi \underline{T}_{i k}+\left(\frac{\chi}{3}\right) \varepsilon_{i k}=\chi\left[\underline{T}_{i k}+\left(\frac{1}{3}\right) \varepsilon_{i k}\right]\right\}_{i k} .
$$

Of which one of the Friedmann's solutions is:

$$
\left\{\left(\frac{\mathrm{d}^{2} R(t)}{\mathrm{d} t^{2}}\right)=-\left(\frac{4 \pi G R}{3}\right)\left(\rho+\frac{3 P}{c^{2}}\right)+\left(\frac{\Lambda}{3}\right) R\right\} .
$$

If now we argue that the pressure of mass-space increase is an additional pressure it follows that the global gravitational acceleration is $g_{\mathrm{tot}}=-g_{\mathrm{grv}}+\left(-g_{\mathrm{gas}}\right)+$ $g_{\text {exp }}$

or

$$
\left\{g_{\mathrm{tot}}=\left(\frac{\mathrm{d} V_{\mathrm{all}}}{\mathrm{d} t}\right)=-\left(\frac{4 \pi G R \rho_{\mathrm{mat}}}{3}\right)+\left(-\frac{4 \pi G R P_{\mathrm{gal}}}{c^{2}}\right)+\left(\frac{4 \pi G R P_{\text {exp }}}{c^{2}}\right)\right\} .
$$

Adding the pressure of increasing space we obtain an "outward" fall acceleration as if there is an antigravity action $g \rightarrow\left(+g_{\text {exp }}\right)$. In the literature it is introduced the repulsive energy of the "vacuum" which is opposed to gravity and to which it is associated a mass density at rest $\left(\rho_{V}\right)$ and a pressure $\left.\left[P_{V}=-(1 / 3) \rho_{V} c^{2}\right)\right]$. Then, in literature it is

$$
\left\{g_{\mathrm{tot}}=\left(\frac{\mathrm{d} V_{\mathrm{all}}}{\mathrm{d} t}\right)=-\left(\frac{4 \pi G R}{3}\right)\left(\rho_{\mathrm{mat}}-\rho_{v}-\frac{3 P_{v}}{c^{2}}\right)\right\}
$$

where we overlooked the pressure galactic. Here the pressure $P_{V}$ and the mass $\left(\rho_{V}\right)$ generate "anti-gravity". All this derives from putting $\left[T_{i k}=0\right]$ and from connecting of $\Lambda$ to energy of the vacuum.

However, if we impose that always it is [ $T_{i k} \neq 0$ ], then we cannot associate to $\Lambda$ the energy and pressure of the "quantum" vacuum.

Instead in the case $\left[T_{i k} \neq 0\right]$ we have that $\left(P_{\exp }\right) \Leftrightarrow\left(\varepsilon_{\text {exp }}\right) \equiv\left(\varepsilon_{\mathrm{de}}\right)$, where $\left(\varepsilon_{\mathrm{de}}\right)$ is correlated to the mass-space flow even if it is interpreted in the literature as "dark energy". We add also that in this model (in which the mass creates the space) the contribution in energy of the quantum vacuum to the gravity, expressed in quantity of mass at rest, is null. 
We note firstly that base field $\left(\Xi_{U}\right)$ does not contain any additional transversal coupling that provides mass (and thus define the space); then it could express a lattice with "flat" structure, if conceded that we can talk about space in it. Therefore, the vacuum state of $\left(\Xi_{U}\right)$, as they are of it the virtual photons, it is cannot give any contribution to gravity. Recall that in the massive fields (see chains $\Xi_{i}$ ) the mass is associated with the frequency relative to the transversal coupling which realizes the lattice of IQuO chains, independently to having quanta full or empty.

It following that one only $\left(\underline{\boldsymbol{\Xi}}_{i}\right)$ lattice-chain associated with a single massive particle, originated in $\left(\Xi_{U}\right)$, will be to "metric" flat while a superposition of massive lattices can generate an additional elastic tensions that "curves" the resulting lattice. Yet we also note that an only pair (virtual and not observed) can be expressed as a single massive lattice because it is originated from a single lattice $\left(\Xi_{i}\right)$ massless (a virtual photon). It follows that the lattices of virtual massive pairs cannot express "curvature" (see Figure 3 ) and then no gravitational field. In this cosmologic model the contribution of the quantum vacuum to gravity is null. Then we cannot longer accept the term $\left(\rho_{V}\right)$ in Equation (5.67) and $\left(P_{V}\right)$, which instead is replaced by $\left(P_{\exp }\right) \Leftrightarrow\left[\left(\varepsilon_{\text {exp }}\right) \equiv\left(\varepsilon_{\mathrm{de}}\right)\right]$. Instead, in our opinion, the mass flow, corresponding to the space flow generating expansion, would determine only values of mass density, referred as dark mass and as to ordinary matter; then we would have to consider the contribution of this in gravity or $\left(\rho_{v}\right) \geq\left(\rho_{\mathrm{dm}}\right)$ and $\left(\rho_{\mathrm{m}}\right)$ $\geq\left(\rho_{\mathrm{om}}, \rho_{\mathrm{dm}}\right)$, where $\left(\rho_{\mathrm{om}}\right)$ is the density of ordinary matter:

$$
\left\{g_{\mathrm{tot}}=\left(\frac{\mathrm{d} V_{\mathrm{all}}}{\mathrm{d} t}\right)=-\left(\frac{4 \pi G R}{3}\right)\left(\rho_{\mathrm{mat}}+\rho_{\mathrm{dm}}-\frac{3 P_{\text {exp }}}{c^{2}}\right)\right\}
$$

\section{Conclusions}

It is evident in this paper that the idea of the universe structured in fundamental terms on a basic Field $\Xi_{U} \equiv(\Xi, \Xi)$, doesn't oppose to the actual model of universe. Rather it tries to resolve some problem list leading and it opens the way toward new horizons on the birth and evolution of the universe. By this new approach we have tried to face the fundamental problem of the expansion of the universe, explaining it as "creation" of space in the USTF. The idea which sees the mass as generating of space completes the theory of the general relativity: the mass itself acts on the space in totus, because it curves the space and creates it. Besides the mass-space equivalence introduces a variable metric universe that clarifies some cosmological problem list not still resolved:

- The problem of Universe expansion problem described only mathematically and not physically by scale parameter $a(t)$ associated to galactic distances.

- The Dark Mass problem.

- Expansive acceleration whose cause is dark energy.

- The unconvincing hypothesis that the universe became at flat metric during the inflation (change of metric in an "isolated" universe). 
- The Higgs field could be associated to massive scalar field $\Xi_{U}$.

The explanatory theses of this innovative cosmological theory are remarkable:

- The physical explanation of the expansion as the effect of creating "space".

- The creation of mass implies the creation of space: Space-Mass equivalence.

- The existence of an underground system physical with structure of not field $(\Theta)$ which exchanges mass-energy with the universe.

- The acceleration of galaxies as consequence of the pressure derived from the flow of mass-space in the fund lattice $\left(\Xi_{U}\right)$.

- The Einstein's parameter cosmological $(\Lambda)$ is not more ad hoc postulate but it proceeds directly from the pressure of the incoming space flow into universe $U$.

- The dark energy is coincident with the flow of quanta from $(\Theta)$ to $U$.

- In this paper one solves the question of coincidence $\left(\Omega_{\mathrm{dm}} / \Omega_{\mathrm{de}} \approx 1\right)$ thanks at equivalence mass-flow $\Leftrightarrow$ space flow.

- The variable metric involves that the universe can go through phases to different curvature.

The cosmologic model with mass increasing describes a universe which itself is initially developed with variable open metric because the $(\Theta)$ origins a mass-space flow (see the expansion) and an additional pressure in order to increasing space. Because the observations point on Universe at flat metric, one can conjecture that the mass increases more that the space; one can believe that the universe has reaching the flat phase after once of 14.82 billions of years from the BB; we think that the Universe after the flat phase will pass to a metrics closed. Then there will be prevalence of the gravitational force which will actually will determine a deceleration of the expansion until the stop for after begin a phase of contraction. So it is admitted the possibility that to end of this phase of contraction there will is a "Big Crunch".

Besides, to obtain therefore a variable metric universe one must assume the existence of a flow of quanta from the not field $(\Theta)$ to universe U. Having admitted $(\Theta)$-underground it is necessary to found the gateway of the mass-space flow which increases the Universe. A possible gateway of Mass-Space flow which origins the dark matter can be individuated in Black Holes, while for ordinary matter it is necessary admit a global diffusion from $(\Theta)$ to $\mathrm{U}$ through the $\left(\underline{\underline{\Xi}}_{\mathrm{U}}\right)$-field, always present in all interactions between particles.

If we assert that the mass-space flow gives rise also to the field of dark mass enveloping each galaxy, we are led to suspect that the "hole", from which emerges the flow, is located in the center of every galaxy. However it is known [?] that in the centre of each galaxy there is a black hole $(\mathrm{BH})$, therefore we should place the "aperture valve", that allows the passage of quanta from $(\Theta)$ in $\left(\underline{\Xi}_{D}\right)$, just inside of a black hole and also to admit that the field of dark mass comes out from it. It is the same thing to say that the source of the dark mass is a black hole.

This conjecture is possible only we admit that $(\Theta)$ is a physical system at not-field structure with the possibility to exist inside $\mathrm{BH}$ and to emerge from a closed gra- 
vitational field as that one of $\mathrm{BH}$.

In conclusion the completing the relativistic cosmology introduces new hypothesis on the origin the matter dark and energy as on the whole universe; not only but it opens the way toward a new theory which can explorer also the Black Holes.

Nevertheless we cannot avoid of cite that the cosmological problems like the dark energy and dark matter find solution through extended theories of gravity. Really it is possible note that this cosmological theory certainty belongs to group of theories which extend the Einstein's gravity because it attempt of resolving cosmological problems "extending" the relation between space and mass: the result is remarkable because we obtain solution to fundamental problems and new information about some important aspects as "space and energy" and vacuum. A fundamental paper on this issue is Corda, Christian, Int. J. Mod. Phys. D, 18, 2275 (2009).

\section{Acknowledgements}

We would like to thank Dr. Christian Corda for his suggestions and advice in the preparation of the paper.

\section{References}

[1] Guido, G. and Filippelli, G. (2017) The Universe at Lattice-Fields. Journal of High Energy Physics, Gravitation and Cosmology, 3, 828-860. https://doi.org/10.4236/jhepgc.2017.34060

[2] Guido, G. (2012) The Substructure of a Quantum Oscillator Field. https://arxiv.org/abs/1208.0948

[3] Guido, G. (2014) The Substructure of a Quantum Field-Oscillator. Hadronic Journal, 37, 83.

[4] Einstein, A. (1923) The Meaning of Relativity. Princeton University Press, Princeton.

[5] Zel'Dovich, Ya.B. and Novikov, I.D. (1983) Relativistic Astrophysics. vol. 2: The Structure and Evolution of the Universe. The University of Chicago Press, Chicago.

[6] Friedmann, A. (1922) Über die krmmung des raumes. Zeitschrift für Physik, 10, 377-386. https://doi.org/10.1007/BF01332580

[7] Perlmutter, S., et al. (1998) Discovery of a Supernova Explosion at Half the Age of the Universe. Nature, 391, 51-54. https://doi.org/10.1038/34124

[8] Corda, C. (2009) Interferometric Detection of Gravitational Waves: The Definitive Test for General Relativity. International Journal of Modern Physics D, 18, 2275. https://doi.org/10.1142/S0218271809015904

[9] Poggianti, B., et al. (2017) Ram Pressure Feeding Supermassive Black Holes. Nature, 548, 304-309. https://doi.org/10.1038/nature23462

[10] Landau, L.D. and Lifšits, E.M. (1976) Teoria dei campi. Editori Riuniti Press, Rome, 339-363. 